\title{
GEOMETRY OF THE MADELUNG TRANSFORM
}

\author{
BORIS KHESIN, GERARD MISIOŁEK, AND KLAS MODIN
}

\begin{abstract}
The Madelung transform is known to relate Schrödingertype equations in quantum mechanics and the Euler equations for barotropic-type fluids. We prove that, more generally, the Madelung transform is a Kähler map (i.e. a symplectomorphism and an isometry) between the space of wave functions and the cotangent bundle to the density space equipped with the Fubini-Study metric and the Fisher-Rao information metric, respectively. We also show that Fusca's momentum map property of the Madelung transform is a manifestation of the general approach via reduction for semi-direct product groups. Furthermore, the Hasimoto transform for the binormal equation turns out to be the $1 \mathrm{D}$ case of the Madelung transform, while its higher-dimensional version is related to the problem of conservation of the Willmore energy in binormal flows.
\end{abstract}

\section{Contents}

1. Introduction

2. Madelung transform as a symplectomorphism 4

2.1. Symplectic properties

2.2. Example: linear and nonlinear Schrödinger equations $\quad 7$

2.3. Madelung transform as a Hasimoto map in 1D 9

3. Madelung transform as an isometry of Kähler manifolds 13

3.1. Metric properties 13

3.2. Geodesics of the Sasaki-Fisher-Rao metric 14

3.3. Example: 2-component Hunter-Saxton equation $\quad 15$

4. Madelung transform as a momentum map 16

$\begin{array}{lll}\text { 4.1. A group action on the space of wave functions } & 17\end{array}$

4.2. The inverse of the Madelung transform 18

4.3. A reminder on momentum maps 19

4.4. Madelung transform is a momentum map 19

4.5. Multi-component Madelung transform as a momentum map 21

4.6. Example: general compressible fluids 22

4.7. Geometry of semi-direct product reduction 23

Appendix A. The functional-analytic setting 24

Appendix B. A comment on rescaling constants 25

$\begin{array}{ll}\text { References } & 26\end{array}$ 


\section{INTRODUCTION}

In 1927 E. Madelung [14] introduced a transformation, which now bears his name, in order to give an alternative formulation of the linear Schrödinger equation for a single particle moving in an electric field as a system of equations describing the motion of a compressible inviscid fluid. Since then other derivations have been proposed in the physics literature primarily in connection with various models in quantum hydrodynamics and optimal transport, cf. $[16,20,15]$.

In this paper we focus on the geometric aspects of Madelung's construction and prove that the Madelung transform possesses a number of surprising properties. It turns out that in the right setting it can be viewed as a symplectomorphism, an isometry, a Kähler morphism or a generalized Hasimoto map. Furthermore, geometric properties of the Madelung transform are best understood not in the setting of the $L^{2}$-Wasserstein geometry but (an infinite-dimensional analogue of) the Fisher-Rao information geometry - the canonical Riemannian geometry of the space of probability densities. These results can be summarized in the following theorem (a joint version of Theorems 2.4 and 3.3 below).

Main Theorem. The Madelung transform is a Kähler morphism between the cotangent bundle of the space of smooth probability densities, equipped with the (Sasaki)-Fisher-Rao metric, and an open subset of the infinitedimensional complex projective space of smooth wave functions, equipped with the Fubini-Study metric.

The statement is valid in both the Sobolev topology of $H^{s}$-smooth functions and Fréchet topology of $C^{\infty}$-smooth functions. In a sense the Madelung transform resembles the passage from Euclidean to polar coordinates in the infinite-dimensional space of wave functions, where the modulus is a probability density and the phase corresponds to fluid's vector field. The above theorem shows that, after projectivization, this transform relates not only equations of hydrodynamics and those of quantum physics, but the corresponding symplectic structures underlying them as well. Surprisingly, it also turns out to be an isometry between two well-known Riemannian metrics in geometry and statistics.

This result reveals tighter links between hydrodynamics, quantum information geometry and geometric quantum mechanics. Important in our constructions is a reformulation of Newton's equations on these spaces of diffeomorphisms and probability densities. This reformulation can be viewed as an extension of Arnold's formalism for the Euler equations of ideal hydrodynamics $[1,2]$.

Our first motivation comes from hydrodynamics where groups of diffeomorphisms arise as configuration spaces for flows of compressible and incompressible fluids in a domain $M$ (typically, a compact connected Riemannian manifold with a volume form $\mu$ ). When equipped with a metric given at the 
identity diffeomorphism by the $L^{2}$ inner product (corresponding essentially to the kinetic energy) the geodesics of the group $\operatorname{Diff}(M)$ of smooth diffeomorphisms of $M$ describe motions of the gas of noninteracting particles in $M$ whose velocity field $v$ satisfies the inviscid Burgers equation

$$
\dot{v}+\nabla_{v} v=0 .
$$

On the other hand, when restricted to the subgroup $\operatorname{Diff}_{\mu}(M)$ of volumepreserving diffeomorphisms, the $L^{2}$-metric becomes right-invariant, and its geodesics can be viewed as motions of an ideal (that is, incompressible and inviscid) fluid in $M$ whose velocity field satisfies the incompressible Euler equations

$$
\left\{\begin{array}{l}
\dot{v}+\nabla_{v} v=-\nabla p \\
\operatorname{div} v=0
\end{array}\right.
$$

Here the pressure gradient $\nabla p$ is defined uniquely by the divergence-free condition on the velocity field $v$ and can be viewed as a constraining force on the fluid. What we describe below can be regarded as an extension of this framework to various equations of compressible fluids, where the evolution of density becomes foremost important.

Our second motivation is to study the geometry of the space of densities. Namely, consider the projection $\pi: \operatorname{Diff}(M) \rightarrow \operatorname{Dens}(M)$ of the full diffeomorphism group $\operatorname{Diff}(M)$ onto the space $\operatorname{Dens}(M)$ of normalized smooth densities on $M$. The fiber over a density $\nu$ consists of all diffeomorphisms $\phi$ that push forward the Riemannian volume form $\mu$ to $\nu$, that is, $\phi_{*} \mu=\nu$. It was shown by Otto [17] that $\pi$ is a Riemannian submersion between $\operatorname{Diff}(M)$ equipped with the $L^{2}$-metric and $\operatorname{Dens}(M)$ equipped with the (Kantorovich-Wasserstein) metric used in the optimal mass transport. More interesting for our purposes is that a Riemannian submersion arises also when $\operatorname{Diff}(M)$ is equipped with a right-invariant homogeneous Sobolev $\dot{H}^{1}$-metric and Dens $(M)$ with the Fisher-Rao metric which plays an important role in geometric statistics, see [9].

In the present paper we prove the Kähler property of the Madelung transform thus establishing a close relation of the cotangent space of the space of densities and the projective space of wave functions on $M$. Furthermore, this transform also identifies many Newton-type equations on these spaces that are naturally related to equations of fluid dynamics.

As an additional perspective, the connection between equations of quantum mechanics and hydrodynamics described below might shed some light on the hydrodynamical quantum analogs studied in [5, 4]: the motion of bouncing droplets in certain vibrating liquids manifests many properties of quantum mechanical particles. While bouncing droplets have a dynamical boundary condition with changing topology of the domain every period, apparently a more precise description of the phenomenon should involve a certain averaging procedure for the hydrodynamical system in a periodically 
changing domain. Then the droplet-quantum particle correspondence could be a combination of the averaging and Madelung transform.

Acknowledgements. B.K. is grateful to the IHES in Bures-sur-Yvette and the Weizmann Institute in Rehovot for their support and kind hospitality. B.K. was also partially supported by an NSERC research grant. Part of this work was done while G.M. held the Ulam Chair visiting Professorship in University of Colorado at Boulder. K.M. was supported by EU Horizon 2020 grant No 691070, by the Swedish Foundation for International Cooperation in Research and Higher Eduction (STINT) grant No PT2014-5823, and by the Swedish Research Council (VR) grant No 2017-05040.

\section{MADELUnG TRANSFORM AS A SYMPLECTOMORPHISM}

In this section we show that the Madelung transform induces a symplectomorphism between the cotangent bundle of smooth probability densities and the projective space of smooth non-vanishing complex-valued wave functions.

Definition 2.1. Let $\mu$ be a (reference) volume form on $M$ such that $\int_{M} \mu=$ 1. The space of probability densities on a compact connected oriented $n$ manifold $M$ is

$$
\operatorname{Dens}^{s}(M)=\left\{\rho \in H^{s}(M) \mid \rho>0, \int_{M} \rho \mu=1\right\},
$$

where $H^{s}(M)$ denotes the space of real-valued functions on $M$ of Sobolev class $H^{s}$ with $s>n / 2$ (including the case $s=\infty$ corresponding to $C^{\infty}$ functions). ${ }^{1}$

The space Dens ${ }^{s}(M)$ can be equipped in the standard manner with the structure of a smooth infinite-dimensional manifold (Hilbert, if $s<\infty$ or Fréchet, if $s=\infty)$. It is an open subset of an affine hyperplane in $H^{s}(M)$. Its tangent bundle is trivial

$$
T \operatorname{Dens}^{s}(M)=\operatorname{Dens}^{s}(M) \times H_{0}^{s}(M)
$$

where $H_{0}^{s}(M)=\left\{c \in H^{s}(M) \mid \int_{M} c \mu=0\right\}$. Likewise, the (regular part of the) co-tangent bundle is

$$
T^{*} \operatorname{Dens}^{s}(M)=\operatorname{Dens}^{s}(M) \times H^{s}(M) / \mathbb{R},
$$

where $H^{s}(M) / \mathbb{R}$ is the space of cosets $[\theta]$ of functions $\theta$ modulo additive constants $[\theta]=\{\theta+c \mid c \in \mathbb{R}\}$. The pairing is given by

$$
T_{\rho} \operatorname{Dens}^{s}(M) \times T_{\rho}^{*} \operatorname{Dens}^{s}(M) \ni(\dot{\rho},[\theta]) \mapsto \int_{M} \theta \dot{\rho} \mu .
$$

\footnotetext{
${ }^{1}$ From a geometric point of view it is more natural to define densities as volume forms instead of functions. This way, they become independent of the reference volume form $\mu$. However, since some of the equations studied in this paper depends on the reference volume form $\mu$ anyway, it is easier to define densities as functions to avoid notational overload.
} 


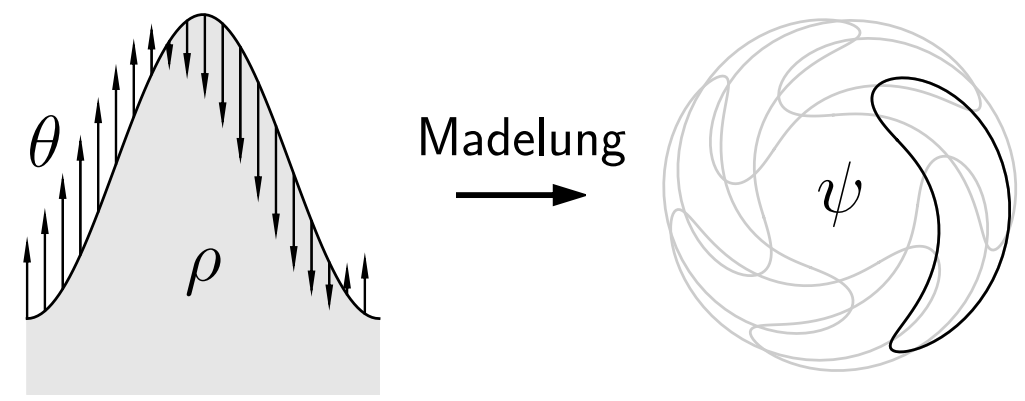

Figure 1. Illustration of the Madelung transform $\mathbf{\Phi}$ on $S^{1}$. For $x \in S^{1}$, a probability density $\rho(x)>0$ and a dual infinitesimal probability density $\theta(x)$ are mapped to a wave function $\psi(x)=\sqrt{\rho \mathrm{e}^{\mathrm{i} \theta}} \in \mathbb{C}$, which is defined up to rigid rotations of the complex plane.

It is independent of the choice of $\theta$ in the coset $[\theta]$ since $\int_{M} \dot{\rho} \mu=0$.

Definition 2.2. The Madelung transform is a map $\boldsymbol{\Phi}$ which to any pair of functions $\rho: M \rightarrow \mathbb{R}_{>0}$ and $\theta: M \rightarrow \mathbb{R}$ associates a complex-valued function

$$
\boldsymbol{\Phi}:(\rho, \theta) \mapsto \psi:=\sqrt{\rho \mathrm{e}^{\mathrm{i} \theta}}=\sqrt{\rho} \mathrm{e}^{\mathrm{i} \theta / 2} .
$$

Remark 2.3. The latter expression defines a particular branch of the square root $\sqrt{\rho \mathrm{e}^{\mathrm{i} \theta}}$. The map $\boldsymbol{\Phi}$ is unramified, since $\rho$ is strictly positive. Note that this map is not injective because $\theta$ and $\theta+4 \pi k$ have the same image. Despite this fact, there is, as we shall see next, a natural geometric setting in which the Madelung transform (2) becomes invertible.

2.1. Symplectic properties. Let $H^{s}(M, \mathbb{C})$ denote the space of complexvalued functions of Sobolev class on a compact connected manifold $M$ and let $\mathbb{P} H^{s}(M, \mathbb{C})$ denote the corresponding complex projective space. Its elements can be represented as cosets of the unit $L^{2}$-sphere of complex functions

$$
[\psi]=\left\{\mathrm{e}^{\mathrm{i} \tau} \psi \mid \psi \in H^{s}(M, \mathbb{C}),\|\psi\|_{L^{2}}=1 \text { and } \tau \in \mathbb{R}\right\} .
$$

If $\tilde{\psi} \in[\psi]$ is nowhere vanishing then every other representative in the coset $[\psi]$ is nowhere vanishing as well. In particular, $\mathbb{P} H^{s}(M, \mathbb{C} \backslash\{0\})$ is an open subset and hence a submanifold of $\mathbb{P} H^{s}(M, \mathbb{C})$.

Theorem 2.4. The Madelung transform (2) induces a map

$$
\mathbf{\Phi}: T^{*} \operatorname{Dens}^{s}(M) \rightarrow \mathbb{P} H^{s}(M, \mathbb{C} \backslash\{0\})
$$

which, up to scaling by 4 , is a symplectomorphism ${ }^{2}$ with respect to the canonical symplectic structure of $T^{*} \operatorname{Dens}^{s}(M)$ and the symplectic form of the Kähler structure on $\mathbb{P} H^{s}(M, \mathbb{C})$.

\footnotetext{
${ }^{2}$ In the Fréchet topology of smooth functions if $s=\infty$.
} 
Proof. We need to establish the following three steps: (i) $\boldsymbol{\Phi}$ is well-defined, (ii) $\boldsymbol{\Phi}$ is smooth, surjective and injective and (iii) $\boldsymbol{\Phi}$ is symplectic.

(i) Let $\rho \in \operatorname{Dens}^{s}(M)$. Recall that the elements of $T_{\rho}^{*} \operatorname{Dens}^{s}(M)$ are cosets of $H^{s}$ functions on $M$ modulo constants and given any $\theta \in H^{s}(M, \mathbb{R})$ and any $\tau \in \mathbb{R}$ the Madelung transform maps $(\rho, \theta+\tau)$ to $\sqrt{\rho} \mathrm{e}^{\mathrm{i}(\theta+\tau) / 2}$. If $s>n / 2$ then standard results on products and compositions of Sobolev functions (cf. e.g., [18]) show that it is smooth as a map to $H^{s}(M, \mathbb{C})$. Furthermore, we have

$$
\left\|\sqrt{\rho} \mathrm{e}^{\mathrm{i}(\theta+\tau) / 2}\right\|_{L^{2}}=\left\|\sqrt{\rho} \mathrm{e}^{\mathrm{i} \theta / 2}\right\|_{L^{2}}=\|\sqrt{\rho}\|_{L^{2}}=1
$$

so that that cosets $(\rho,[\theta])$ are mapped to cosets $[\psi]$, i.e., the map is welldefined.

(ii) Surjectivity and smoothness of $\boldsymbol{\Phi}$ are evident. To prove injectivity for the cosets recall that inverting the Madelung map amounts essentially to rewriting of a non-vanishing complex-valued function in polar coordinates. Since preimages for a given $\psi$ differ by a constant polar argument $\tilde{\theta}=$ $\theta+2 \pi k$, they define the same coset $[\theta]$. Similarly, changing $\psi$ by a constant phase does not affect the argument coset $[\theta]$, which implies injectivity of the map between the cosets $(\rho,[\theta])$ and $[\psi] .^{3}$

(iii) The canonical symplectic form on $T^{*} \operatorname{Dens}^{s}(M)$ is given by

$$
\Omega_{(\rho,[\theta])}^{T^{*} \text { Dens }}\left(\left(\dot{\rho}_{1},\left[\dot{\theta}_{1}\right]\right),\left(\dot{\rho}_{2},\left[\dot{\theta}_{2}\right]\right)\right) \mu=\int_{M}\left(\dot{\theta}_{1} \dot{\rho}_{2}-\dot{\theta}_{2} \dot{\rho}_{1}\right) \mu
$$

Since $\int_{M} \dot{\rho}_{k} \mu=0$ it follows that it is well-defined on the cosets $\left[\dot{\theta}_{i}\right]$. The symplectic form on $\mathbb{P} H^{s}(M, \mathbb{C})$ is given by

$$
\Omega_{[\psi]}^{\mathbb{P} H^{s}}\left(\left[\dot{\psi}_{1}\right],\left[\dot{\psi}_{2}\right]\right)=\int_{M} \operatorname{Im}\left(\dot{\psi}_{1} \overline{\dot{\psi}_{2}}\right) \mu
$$

The tangent vectors can be described as cosets $\left[\dot{\psi}_{k}\right]=\left\{\mathrm{i} c \psi+\dot{\psi}_{k} \mid c \in \mathbb{R}\right\}$ obtained by differentiating $[\psi]=\left\{\psi \mathrm{e}^{\mathrm{i} \tau} \mid \tau \in \mathbb{R}\right\}$. One can see that $\Omega^{\mathbb{P} H^{s}}$ is well-defined on the coset vectors which follows from $\int_{M} \operatorname{Re}\left(\psi \dot{\bar{\psi}}_{k}\right) \mu=0$ and a straightforward calculation. Finally, for $\psi=\mathbf{\Phi}(\rho,[\theta])$ the tangent vector

\footnotetext{
${ }^{3}$ Note that the injectivity would not hold for $L^{2}$ functions, or even for smooth functions if $M$ were not connected. Indeed, the arguments of the preimages could then have incompatible integer jumps at different points of $M$. For continuous functions on a connected $M$ it suffices to fix the argument at one point only.
} 
is $T_{(\rho,[\theta])} \boldsymbol{\Phi}(\dot{\rho},[\dot{\theta}])=1 / 2(\dot{\rho} / \rho+\mathrm{i} \dot{\theta}) \boldsymbol{\Phi}(\rho,[\theta])$. Then (5) gives

$$
\begin{aligned}
& \Omega_{\mathbf{\Phi}(\rho,[\theta])}^{\mathbb{P} H^{s}}\left(T_{(\rho,[\theta])} \boldsymbol{\Phi}\left(\dot{\rho}_{1},\left[\dot{\theta}_{1}\right]\right), T_{(\rho,[\theta])} \boldsymbol{\Phi}\left(\dot{\rho}_{2},\left[\dot{\theta}_{2}\right]\right)\right)= \\
& =\frac{1}{4} \int_{M} \operatorname{Im}\left(\left(\frac{\dot{\rho}_{1}}{\rho}+\mathrm{i} \dot{\theta}_{1}\right)\left(\frac{\dot{\rho}_{2}}{\rho}-\mathrm{i} \dot{\theta}_{2}\right) \psi \bar{\psi}\right) \mu= \\
& =\frac{1}{4} \int_{M}\left(\dot{\theta}_{1} \frac{\dot{\rho}_{2}}{\rho}-\dot{\theta}_{2} \frac{\dot{\rho}_{1}}{\rho}\right) \rho \mu=\frac{1}{4} \int_{M}\left(\dot{\theta}_{1} \dot{\rho}_{2}-\dot{\theta}_{2} \dot{\rho}_{1}\right) \mu \\
& =\frac{1}{4} \Omega_{(\rho,[\theta])}^{T^{*} \text { Dens }}\left(\left(\dot{\rho}_{1},\left[\dot{\theta}_{1}\right]\right),\left(\dot{\rho}_{2},\left[\dot{\theta}_{2}\right]\right)\right),
\end{aligned}
$$

which completes the proof.

Remark 2.5. In $\S 4$ the inverse Madelung transform is defined for any $C^{1}$ function with no restriction on strict positivity of $|\psi|^{2}$. It can be defined similarly in a Sobolev setting. Furthermore, extending Fusca [7], we will also show that it can be understood as a momentum map for a natural action of a certain semi-direct product group. Thus the Madelung transform relates the standard symplectic structure on the space of wave functions and the linear Lie-Poisson structure on the corresponding dual Lie algebra.

Remark 2.6. The fact that the Madelung transform is a symplectic submersion between the cotangent bundle of the space of densities and the unit sphere $S^{\infty} \subset H^{s}(M, \mathbb{C} \backslash\{0\})$ of non-vanishing wave functions was proved by von Renesse [20]. The stronger symplectomorphism property proved in Theorem 2.4 is achieved by considering projectivization $\mathbb{P} H^{s}(M, \mathbb{C} \backslash\{0\})$.

2.2. Example: linear and nonlinear Schrödinger equations. Let $\psi$ be a wave function on a Riemannian manifold $M$ and consider the family of Schrödinger (or Gross-Pitaevsky) equations with Planck's constant $\hbar=1$ and mass $m=1 / 2$

$$
\mathrm{i} \dot{\psi}=-\Delta \psi+V \psi+f\left(|\psi|^{2}\right) \psi
$$

where $V: M \rightarrow \mathbb{R}$ and $f:(0, \infty) \rightarrow \mathbb{R}$. If $f \equiv 0$ we obtain the linear Schrödinger equation with potential $V$. If $V \equiv 0$ we obtain the family of non-linear Schrödinger equations (NLS); two typical choices are $f(a)=\kappa a$ and $f(a)=\frac{1}{2}(a-1)^{2}$.

Note that Equation (6) is Hamiltonian with respect to the symplectic structure induced by the complex structure of $L^{2}(M, \mathbb{C})$. Indeed, recall that the real part of a Hermitian inner product defines a Riemannian structure and the imaginary part defines a symplectic structure, so that

$$
\Omega\left(\psi_{1}, \psi_{2}\right):=\operatorname{Im}\left\langle\left\langle\psi_{1}, \psi_{2}\right\rangle\right\rangle_{L^{2}}=\operatorname{Re}\left\langle\left\langle i \psi_{1}, \psi_{2}\right\rangle\right\rangle_{L^{2}}
$$

defines a symplectic form $\Omega$ corresponding to the complex structure $J(\psi)=$ $\mathrm{i} \psi$. The Hamiltonian function for the Schrödinger equation (6) is

$$
H(\psi)=\frac{1}{2}\|\nabla \psi\|_{L^{2}}^{2}+\frac{1}{2} \int_{M}\left(V|\psi|^{2}+F\left(|\psi|^{2}\right)\right) \mu,
$$

where $F:(0, \infty) \rightarrow \mathbb{R}$ is a primitive function of $f$, namely $F^{\prime}=f$. 
Observe that the $L^{2}$-norm of any solution $\psi$ of (6) is conserved in time. Furthermore, the Schrödinger equation is also equivariant with respect to a constant phase shift $\psi(x) \mapsto e^{\mathrm{i} \tau} \psi(x)$ and therefore descends to the projective space $\mathbb{P} H^{s}(M, \mathbb{C})$. It can be viewed as an equation on the complex projective space, a point of view first suggested in [11].

Proposition 2.7 (cf. [14, 20]). The Madelung transform (3) maps the family of Schrödinger equations (6) to the following system on $T^{*} \operatorname{Dens}^{s}(M)$

$$
\left\{\begin{array}{l}
\dot{\theta}+\frac{1}{2}|\nabla \theta|^{2}-\frac{4 \Delta \sqrt{\rho}}{\sqrt{\rho}}+2 V+2 f(\rho)=0, \\
\dot{\rho}+\operatorname{div}(\rho \nabla \theta)=0 .
\end{array}\right.
$$

Equation (8) has a hydrodynamic formulation as an equation for a barotropictype fluid

$$
\left\{\begin{array}{l}
\dot{v}+\nabla_{v} v+\nabla\left(2 V+2 f(\rho)-\frac{4 \Delta \sqrt{\rho}}{\sqrt{\rho}}\right)=0 \\
\dot{\rho}+\operatorname{div}(\rho v)=0
\end{array}\right.
$$

with potential velocity field $v=\nabla \theta$.

Remark 2.8. Note that (8) only makes sense for $\rho>0$, whereas the NLS equation makes sense even when $\rho \geq 0$. In particular, the properties of the Madelung transform imply that if one starts with a wave function such that $|\psi|^{2}>0$ everywhere, then it remains strictly positive for all $t$ for which the solution to equation (8) is defined, since this holds for $\rho=|\psi|^{2}$ by the continuity equation. Thus, $|\psi|^{2}$ can become non-positive only if $v=\nabla \theta$ stops being a $C^{1}$ vector field (so that the continuity equation breaks).

Proof. Since the transformation $(\rho,[\theta]) \mapsto \psi$ is symplectic, it is enough to work out the Hamiltonian (7) expressed in $(\rho,[\theta])$. First, notice that

$$
\nabla \psi=\mathrm{e}^{\mathrm{i} \theta / 2}\left(\nabla \sqrt{\rho}+\frac{\mathrm{i}}{2} \sqrt{\rho} \nabla \theta\right)
$$

so that

$$
\begin{aligned}
\|\nabla \psi\|_{L^{2}}^{2} & =\left\langle\left\langle\nabla \sqrt{\rho}+\frac{\mathrm{i}}{2} \sqrt{\rho} \nabla \theta, \nabla \sqrt{\rho}+\frac{\mathrm{i}}{2} \sqrt{\rho} \nabla \theta\right\rangle\right\rangle_{L^{2}} \\
& =\langle\langle\nabla \sqrt{\rho}, \nabla \sqrt{\rho}\rangle\rangle_{L^{2}}+\frac{1}{4}\langle\langle\rho \nabla \theta, \nabla \theta\rangle\rangle_{L^{2}}
\end{aligned}
$$

Thus, the Hamiltonian on $T^{*} \operatorname{Dens}^{s}(M)$ corresponding to the Schrödinger Hamiltonian (7) is

$$
H(\rho,[\theta])=\frac{1}{2} \int_{M}\left(\frac{1}{4}|\nabla \theta|^{2} \rho+|\nabla \sqrt{\rho}|^{2}\right) \mu+\frac{1}{2} \int_{M}(V \rho+F(\rho)) \mu .
$$

Since

$$
\frac{\delta H}{\delta \rho}=\frac{1}{8}|\nabla \theta|^{2}-\frac{\Delta \sqrt{\rho}}{\sqrt{\rho}}+\frac{1}{2} V+\frac{1}{2} f(\rho) \quad \text { and } \quad \frac{\delta H}{\delta \theta}=-\frac{1}{4} \operatorname{div}(\rho \nabla \theta)
$$


the result now follows from Hamilton's equations

$$
\dot{\theta}=-4 \frac{\delta H}{\delta \rho}, \quad \dot{\rho}=4 \frac{\delta H}{\delta \theta}
$$

for the canonical symplectic form (4) scaled by $1 / 4$.

Corollary 2.9. The Hamiltonian system (8) on $T^{*} \operatorname{Dens}^{s}(M)$ for potential solutions of the barotropic equation (9) is mapped symplectomorphically to the Schrödinger equation (6).

Remark 2.10. Conversely, classical PDE of hydrodynamic type can be expressed as NLS-type equations. For example, potential solutions $v=\nabla \theta$ of the compressible Euler equations of a barotropic fluid are Hamiltonian on $T^{*} \operatorname{Dens}^{s}(M)$ with the Hamiltonian given as the sum of the kinetic energy $K=\frac{1}{2} \int_{M}|\nabla \theta|^{2} \rho \mu$ and the potential energy $U=\int_{M} e(\rho) \rho \mu$, where $e(\rho)$ is the fluid internal energy, see [10]. They can be formulated as an NLS equation with the Hamiltonian

$$
H(\psi)=\frac{1}{2}\|\nabla \psi\|_{L^{2}}^{2}-\frac{1}{2}\|\nabla|\psi|\|_{L^{2}}^{2}+\int_{M} e\left(|\psi|^{2}\right)|\psi|^{2} \mu .
$$

The choice $e=0$ gives a Schrödinger formulation for potential solutions of Burgers' equation, which describe geodesics in the $L^{2}$-type Wasserstein metric on Dens ${ }^{s}(M)$. Thus, the geometric framework links the optimal transport for cost functions with potentials with the compressible Euler equations and the NLS-type equations described above.

2.3. Madelung transform as a Hasimoto map in 1D. The celebrated vortex filament equation

$$
\dot{\gamma}=\gamma^{\prime} \times \gamma^{\prime \prime}
$$

is an evolution equation on a (closed or open) curve $\gamma \subset \mathbb{R}^{3}$, where $\gamma=$ $\gamma(x, t)$ and $\gamma^{\prime}:=\partial \gamma / \partial x$ and $x$ is an arc-length parameter along $\gamma$. (An equivalent binormal form of this equation $\dot{\gamma}=k(x, t) \mathbf{b}$ is valid in any parametrization, where $\mathbf{b}=\mathbf{t} \times \mathbf{n}$ is the binormal unit vector to the curve at a point $x, \mathbf{t}$ and $\mathbf{n}$ are, respectively, the unit tangent and the normal vectors and $k(x, t)$ is the curvature of the curve at the point $x$ at moment $t$ ). This equation describes a localized induction approximation of the 3D Euler equation of an ideal fluid in $\mathbb{R}^{3}$, where the vorticity of the initial velocity field is supported on a curve $\gamma$. (Note that the corresponding evolution of the vorticity is given by the hydrodynamical Euler equation, which becomes nonlocal in terms of vorticity. By considering the ansatz that keeps only local terms, it reduces to the filament equation above.)

The vortex filament equation is known to be Hamiltonian with respect to the Marsden-Weinstein symplectic structure on the space of curves in $\mathbb{R}^{3}$ and with Hamiltonian given by the length functional, see, e.g., [2]. 


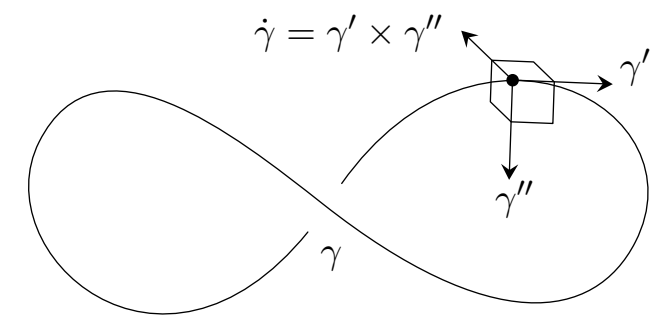

Figure 2. Vortex filament flow: each point of the curve $\gamma$ moves in the direction of the binormal. If $k(x)$ and $\tau(x)$ are the curvature and torsion at $\gamma(x)$, then the wave function $\psi(x)=k(x) \mathrm{e}^{\mathrm{i} \int_{x_{0}}^{x} \tau(\tilde{x}) \mathrm{d} \tilde{x}}$ satisfies the NLS equation. Moreover, the pair of functions $v=2 \tau$ and $\rho=k^{2}$ satisfies the equation of the 1D barotropic fluid, which is a manifestation of the $1 \mathrm{D}$ Madelung transform.

Definition 2.11. The Marsden-Weinstein symplectic structure $\Omega^{M W}$ assigns to a pair of two variations $V, W$ of a curve $\gamma$ (understood as vector fields on $\left.\gamma \subset \mathbb{R}^{3}\right)$ the value $\Omega^{M W}(V, W):=\int_{\gamma} i_{V} i_{W} \mu$, where $\mu$ is the Euclidean volume form in $\mathbb{R}^{3}$.

It turns out that the vortex filament equation becomes the equation of the 1D barotropic-type fluid (9) with $\rho=k^{2}$ and $v=2 \tau$, where $k$ and $\tau$ denote curvature and torsion of the curve $\gamma$, respectively.

In 1972 Hasimoto [8] introduced the following surprising transformation.

Definition 2.12. The Hasimoto transformation assigns to a curve $\gamma$, with curvature $k$ and torsion $\tau$, a wave function $\psi$ according to the formula

$$
(k(x), \tau(x)) \mapsto \psi(x)=k(x) e^{\mathrm{i} \int_{x_{0}}^{x} \tau(\tilde{x}) \mathrm{d} \tilde{x}} .
$$

This map takes the vortex filament equation to the 1D NLS equation $\mathrm{i} \dot{\psi}+\psi^{\prime \prime}+\frac{1}{2}|\psi|^{2} \psi=0$. (A change of the initial point $x_{0}$ in $\int_{x_{0}}^{x} \tau(\tilde{x}) d \tilde{x}$ leads to a multiplication of $\psi(x)$ by an irrelevant constant phase $\left.e^{i \alpha}\right)$. In particular, the filament equation becomes a completely integrable system whose first integrals are obtained by pulling back those of the NLS equation. The first integrals for the filament equation can be written in terms of the total length $\int d x$, the torsion $\int \tau \mathrm{d} x$, the squared curvature $\int k^{2} \mathrm{~d} x$, followed by $\int \tau k^{2} \mathrm{~d} x$ etc.

Remark 2.13. Each of the three forms of the above equations has a natural symplectic or Poisson structure: the Marsden-Weinstein symplectic structure on nonparametrized curves $\{\gamma\}$ for the binormal equation, the linear Lie-Poisson structure on (the dual of) the semidirect product $\mathfrak{s}=$ $\mathfrak{X}\left(S^{1}\right) \ltimes H^{s}\left(S^{1}\right) \ni(v, \rho)$ for the $1 \mathrm{D}$ compressible Euler equation on $v=2 \tau$ and $\rho=k^{2}$, and the standard constant symplectic structure on wave functions $\{\psi\}$ for the NLS. 
Langer and Perline [12] established symplectic properties of the Hasimoto transform. It turns out that the Marsden-Weinstein symplectic structure expressed in terms of the curvature and torsion is mapped by the Hasimoto transform to the constant symplectic structure on wave functions. (The original statement in [12] is more complicated, since the passage from the curve $\gamma$ to its curvature and torsion requires taking two extra derivatives.) This symplectic property has the following heuristic explanation. The MarsdenWeinstein symplectic structure $\Omega^{M W}$ on curves in $\mathbb{R}^{3}$ is, essentially, averaging of the standard symplectic structures in all normal planes to the curve $\gamma$. Furthermore, one can regard the curvature magnitude $|k(x)|$ as the radial coordinate in each normal plane, while (twice) the integral of torsion $\theta(x)=2 \int \tau(x) \mathrm{d} x$ as the angular coordinate (since torsion is by definition the angular velocity of the rotation of the normal vector). This means that the passage from affine coordinates in normal planes to the polar ones $\left(k^{2}, \theta / 2\right)$ is a symplectic map: $d x \wedge d y=(1 / 2) d\left(k^{2}\right) \wedge d \theta$. On the other hand, $k$ and $\theta$ are (adjusted) polar coordinates of the wave function $\psi(x)=k(x) e^{\mathrm{i} \theta(x)}$. So one arrives at the standard symplectic structure on the wave functions, regarded as complex-valued functions.

The following proposition relates the Hasimoto transform to the classical Madelung transform, see Section 2.

Proposition 2.14. The Hasimoto transformation is the Madelung transform in the $1 D$ case.

This can be seen by comparing Definitions 2.2 and 2.12 which make the Hasimoto transform seem much less surprising. Alternatively, one may note that for $\psi(x)=\sqrt{\rho(x)} e^{\mathrm{i} \theta(x) / 2}$ the pair $(\rho, v)$ with $v=\nabla \theta$ satisfies the compressible Euler equation, while in the one-dimensional case these variables are expressed via the curvature $\sqrt{\rho}=\sqrt{k^{2}}=k$ and the (indefinite) integral of torsion $\theta(x) / 2=\int v(x) d x=\int \tau(x) d x$.

Remark 2.15. The filament equation has a higher-dimensional analog for membranes (i.e., compact oriented surfaces $\Sigma$ of co-dimension 2 in $\mathbb{R}^{n}$ ) as a skew-mean-curvature flow $\dot{q}=\mathbf{J}(\mathbf{M C}(q))$, where $q \in \Sigma$ is any point of the membrane, $\mathbf{M C}(q)$ is the mean curvature vector to $\Sigma$ at the point $q$ and $\mathbf{J}$ is the operator of rotation by $\pi / 2$ in the positive direction in every normal space to $\Sigma$. This equation is again Hamiltonian with respect to the Marsden-Weinstein structure $\Omega^{M W}$ on membranes of co-dimension 2 and with a Hamiltonian function given by the $(n-2)$-dimensional volume of the membrane, see e.g. [19].

An intriguing problem in this area is the following.

Question 2.16. Find an analogue of the Hasimoto map, which sends a skew-mean-curvature flow to an NLS-type equation for any $n$. 
The existence of the Madelung transform and its symplectic property in any dimension is a strong indication that such an analog should exist. Indeed, in any dimension by means of the Madelung transform one can pass from the wave function evolved according to an NLS-type equation to the polar form of $\psi$, i.e. to its magnitude $\sqrt{\rho}$ and the phase $\theta$, so that the pair $(\rho, v)$ with $v=\nabla \theta$ will evolve according to the compressible Euler equation. Thus for a surface $\Sigma$ of co-dimension 2 moving according to the skew-meancurvature flow, the problem boils down to interpreting the corresponding characteristics $(\rho, \nabla \theta)$ similarly to the one-dimensional curvature and torsion. (Note that both the pair $(\rho, \theta)$ and the co-dimension 2 surface $\Sigma$ in $\mathbb{R}^{n}$ can be encoded by two functions of $n-2$ variables).

In any dimension the square of the mean curvature vector can be regarded as a natural analog of the density $\rho=\|\mathbf{M C}\|^{2}$. In this case an analog of the total mass of the fluid, i.e. $\int_{\Sigma} \rho \mathrm{d} \sigma$, is the Willmore en$\operatorname{ergy} \mathcal{W}(\Sigma)=\int_{\Sigma}\|\mathbf{M C}\|^{2} \mathrm{~d} \sigma$. An intermediate step in finding a higherdimensional Hasimoto map is then the following

Conjecture 2.17. For a co-dimension 2 surface $\Sigma \in \mathbb{R}^{n}$ moving by the skew-mean curvature flow $\dot{q}=\mathbf{J}(\mathbf{M C}(q))$ the following equivalent properties hold:

i) its Willmore energy $\mathcal{W}(\Sigma)$ is invariant,

ii) its square mean curvature $\rho=\|\mathbf{M C}\|^{2}$ evolves according to the continuity equation $\dot{\rho}+\operatorname{div}(\rho v)=0$ for some vector field $v$ on $\Sigma$.

The equivalence of the two statements is a consequence of Moser's theorem: if the total mass on a surface is preserved, the corresponding evolution of density can be realized as a flow of a time-dependent vector field.

Proposition 2.18. The conjecture is true in dimension 1.

Proof. In 1D the conservation of the Willmore energy is the time invariance of the integral $\mathcal{W}(\gamma)=\int_{\gamma} k^{2} \mathrm{~d} x$ or, equivalently, in the arc-length parameterization, of the integral $\int_{\gamma}\left|\gamma^{\prime \prime}\right|^{2} \mathrm{~d} x$. The latter invariance follows from the following straightforward computation

$$
\begin{aligned}
\frac{d}{d t} \mathcal{W}(\gamma) & =2 \int_{\gamma}\left(\dot{\gamma}^{\prime \prime}, \gamma^{\prime \prime}\right) \mathrm{d} x=-2 \int_{\gamma}\left(\dot{\gamma}^{\prime}, \gamma^{\prime \prime \prime}\right) \mathrm{d} x \\
& =-2 \int_{\gamma}\left(\left(\gamma^{\prime} \times \gamma^{\prime \prime}\right)^{\prime}, \gamma^{\prime \prime \prime}\right) \mathrm{d} x=0 .
\end{aligned}
$$

It would be very interesting to find a higher-dimensional analog of the torsion $\tau$ for co-dimension 2 membranes. Note that the integral of the torsion has to play the role of an angular coordinate in the tangent spaces to $\Sigma$. The torsion would be the gradient part of the field $v$ transporting the density $\rho=\|\mathbf{M C}\|^{2}$. Essentially, the question is how to encode a codimension 2 surface by its mean curvature and torsion. Presumably $\nabla \theta$ as 
an analog of $\tau$ can be regarded as an angle of rotation ("the phase") of the vector $\mathbf{M C}$, i.e. it might play a role of an exact 1-form.

Question 2.19. Is such a surface $\Sigma \subset \mathbb{R}^{n}$ of co-dimension 2 reconstructable (modulo isometries) from the vectors $\mathbf{M C}$, i.e. from their magnitude $\|\mathbf{M C}\|$ and an "angle of rotation", an exact 1-form $d \theta$ ?

Finally, note that such a higher-dimensional Hasimoto map should inherit the Poisson properties of the Madelung transform. The heuristic argument of Remark 2.13 concerning the relation of the symplectic structure in the two-dimensional normal bundle and the space of wave functions should work in any dimension. The Madelung transform between complex-valued wave functions and pairs consisting of densities and gradient potentials has been already shown to be symplectic, see Section 2.2.

\section{MADELUnG TRANSFORM AS AN ISOMETRY OF KÄHLER MANIFOLDS}

3.1. Metric properties. In this section we prove that the Madelung transform is an isometry and a Kähler map between the lifted Fisher-Rao metric on the cotangent bundle $T^{*} \operatorname{Dens}^{s}(M)$ and the Kähler structure corresponding to the Fubini-Study metric on the infinite-dimensional projective space $\mathbb{P} H^{s}(M, \mathbb{C})$.

Definition 3.1. The Fisher-Rao metric on the density space $\operatorname{Dens}^{s}(M)$ is given by

$$
\mathrm{G}_{\rho}(\dot{\rho}, \dot{\rho})=\frac{1}{4} \int_{M} \frac{\dot{\rho}^{2}}{\rho} \mu .
$$

This metric is invariant under the action of the diffeomorphism group. It is, in fact, the only Riemannian metric on $\operatorname{Dens}^{s}(M)$ with this property, cf. e.g., [3].

Next, observe that an element of $T T^{*} \operatorname{Dens}^{s}(M)$ is a 4 -tuple $(\rho,[\theta], \dot{\rho}, \dot{\theta})$, where $\rho \in \operatorname{Dens}^{s}(M),[\theta] \in H^{s}(M) / \mathbb{R}, \dot{\rho} \in H_{0}^{s}(M)$ and $\dot{\theta} \in H^{s}(M)$ subject to the constraint

$$
\int_{M} \dot{\theta} \rho \mu=0
$$

Definition 3.2. The lift of the Fisher-Rao metric to the cotangent bundle $T^{*} \operatorname{Dens}^{s}(M)$ has the form

$$
\mathrm{G}_{(\rho,[\theta])}^{*}((\dot{\rho}, \dot{\theta}),(\dot{\rho}, \dot{\theta}))=\frac{1}{4} \int_{M}\left(\frac{\dot{\rho}^{2}}{\rho}+\dot{\theta}^{2} \rho\right) \mu .
$$

We will refer to this metric as the Sasaki-Fisher-Rao metric.

Next, recall that the canonical (weak) Fubini-Study metric on the complex projective space $\mathbb{P} H^{s}(M, \mathbb{C}) \subset \mathbb{P} L^{2}(M, \mathbb{C})$ is given by

$$
\mathrm{FS}_{\psi}(\dot{\psi}, \dot{\psi})=\frac{\langle\langle\dot{\psi}, \dot{\psi}\rangle\rangle_{L^{2}}}{\langle\langle\psi, \psi\rangle\rangle_{L^{2}}}-\frac{\langle\langle\psi, \dot{\psi}\rangle\rangle_{L^{2}}\langle\langle\dot{\psi}, \psi\rangle\rangle_{L^{2}}}{\langle\langle\psi, \psi\rangle\rangle_{L^{2}}^{2}} .
$$


Theorem 3.3. The Madelung transform $\Phi: T^{*} \operatorname{Dens}^{s}(M) \rightarrow \mathbb{P} H^{s}(M, \mathbb{C})$ is an isometry with respect to the Sasaki-Fisher-Rao metric (15) on $T^{*} \operatorname{Dens}^{s}(M)$ and the Fubini-Study metric (16) on $\mathbb{P} H^{s}(M, \mathbb{C} \backslash\{0\})$.

Proof. We have

$$
T_{(\rho,[\theta])} \boldsymbol{\Phi}(\dot{\rho}, \dot{\theta})=\frac{\dot{\rho}}{2 \sqrt{\rho}} e^{\mathrm{i} \theta / 2}+\frac{\mathrm{i} \dot{\theta} \sqrt{\rho}}{2} e^{\mathrm{i} \theta / 2}=\frac{1}{2}\left(\frac{\dot{\rho}}{\rho}+\mathrm{i} \dot{\theta}\right) \psi
$$

where $\psi=\mathbf{\Phi}(\rho,[\theta])$. Since $\|\psi\|_{L^{2}}^{2}=1$, setting $\dot{\psi}=T_{(\rho, \theta)} \mathbf{\Phi}(\dot{\rho}, \dot{\theta})$ we obtain

$$
\mathrm{FS}_{\psi}(\dot{\psi}, \dot{\psi})=\langle\langle\dot{\psi}, \dot{\psi}\rangle\rangle_{L^{2}}-\langle\langle\dot{\psi}, \psi\rangle\rangle_{L^{2}}\langle\langle\psi, \dot{\psi}\rangle\rangle_{L^{2}}
$$

where

$$
\langle\langle\dot{\psi}, \dot{\psi}\rangle\rangle_{L^{2}}=\frac{1}{4} \int_{M}\left|\frac{\dot{\rho}}{\rho}+\mathrm{i} \theta\right|^{2} \rho \mu=\frac{1}{4} \int_{M}\left(\frac{\dot{\rho}^{2}}{\rho^{2}}+\dot{\theta}^{2}\right) \rho=\mathrm{G}_{(\rho, \theta)}^{*}(\dot{\rho}, \dot{\theta})
$$

and

$$
\langle\langle\dot{\psi}, \psi\rangle\rangle_{L^{2}}=\frac{1}{2} \int_{M}\left(\frac{\dot{\rho}}{\rho}+\mathrm{i} \dot{\theta}\right) \rho \mu=\frac{1}{2} \int_{M} \dot{\rho} \mu+\frac{\mathrm{i}}{2} \int_{M} \dot{\theta} \rho \mu=0,
$$

which proves the theorem.

The metric property in Theorem 3.3 combined with the symplectic property in Theorem 2.4 yields the following.

Corollary 3.4. The cotangent bundle $T^{*} \operatorname{Dens}^{s}(M)$ is a Kähler manifold with the Sasaki-Fisher-Rao metric (15) and the canonical symplectic structure (4) scaled by $1 / 4$. The corresponding integrable almost complex structure is given by

$$
J_{(\rho,[\theta])}(\dot{\rho}, \dot{\theta})=\left(\dot{\theta} \rho,-\frac{\dot{\rho}}{\rho}\right) .
$$

This result can be compared with the result of Molitor [15] who described a similar construction using (the cotangent lift of) the $L^{2}$ Wasserstein metric in optimal transport but obtained an almost complex structure on $T^{*} \operatorname{Dens}^{s}(M)$ which is not integrable. It appears that the Fisher-Rao metric is a more natural choice for such constructions: its lift to $T^{*} \operatorname{Dens}^{s}(M)$ admits a compatible complex (and Kähler) structure. It would be interesting to write down Kähler potentials for all metrics compatible with (17) and identify which of these are invariant under the action of the diffeomorphism group.

3.2. Geodesics of the Sasaki-Fisher-Rao metric. As an isometry the Madelung transform maps geodesics of the Sasaki metric to geodesics of the Fubini-Study metric. The latter are projective lines in the projective space of wave functions. To see which submanifolds are mapped to projective lines by the Madelung transform we need to describe geodesics of the SasakiFisher-Rao metric. 
Proposition 3.5. Geodesics of the Sasaki-Fisher-Rao metric (15) on the cotangent bundle $T^{*} \operatorname{Dens}^{s}(M)$ satisfy the system

$$
\left\{\begin{array}{l}
\frac{\mathrm{d}}{\mathrm{d} t}\left(\frac{\dot{\rho}}{\rho}\right)=-\frac{1}{2}\left(\frac{\dot{\rho}}{\rho}\right)^{2}+\frac{\dot{\theta}^{2}}{2}, \\
\frac{\mathrm{d}}{\mathrm{d} t}(\dot{\theta} \rho)=0 .
\end{array}\right.
$$

Proof. The Lagrangian is given by the metric $L(\rho, \theta, \dot{\rho}, \dot{\theta})=\mathrm{G}_{(\rho, \theta)}^{*}((\dot{\rho}, \dot{\theta}),(\dot{\rho}, \dot{\theta}))$. The variational derivatives are obtained from the formulas

$$
\begin{array}{ll}
\frac{\delta L}{\delta \dot{\rho}}=\frac{1}{2} \frac{\dot{\rho}}{\rho}, & \frac{\delta L}{\delta \dot{\theta}}=\frac{1}{2} \dot{\theta} \rho, \\
\frac{\delta L}{\delta \rho}=-\frac{1}{4}\left(\frac{\dot{\rho}}{\rho}\right)^{2}+\frac{1}{4} \dot{\theta}^{2}, & \frac{\delta L}{\delta \theta}=0,
\end{array}
$$

which yield the equations of motion as stated.

Remark 3.6. The natural projection $(\rho,[\theta]) \mapsto \rho$ is a Riemannian submersion between $T^{*} \operatorname{Dens}^{s}(M)$ equipped with the Sasaki-Fisher-Rao metric (15) and $\operatorname{Dens}^{s}(M)$ equipped with the Fisher-Rao metric (13). The corresponding horizontal distribution on $T^{*} \operatorname{Dens}^{s}(M)$ is given by

$$
\operatorname{Hor}_{(\rho,[\theta])}=\left\{(\dot{\rho}, \dot{\theta}) \in T_{(\rho,[\theta])} \operatorname{Dens}^{s}(M) \mid \dot{\theta}=0\right\} .
$$

Indeed, if $\dot{\theta}=0$ then the equations of motion of Proposition 3.5, restricted to $(\rho, \dot{\rho})$, yield the geodesic equations for the Fisher-Rao metric. One can think of this as a zero-momentum symplectic reduction corresponding to the abelian gauge symmetry $(\rho,[\theta]) \mapsto(\rho,[\theta+f])$ for any function $f \in H^{s}(M)$.

3.3. Example: 2-component Hunter-Saxton equation. This is a system of two equations

$$
\left\{\begin{array}{l}
\dot{v}^{\prime \prime}=-2 v^{\prime} v^{\prime \prime}-v v^{\prime \prime \prime}+\sigma \sigma^{\prime} \\
\dot{\sigma}=-(\sigma v)^{\prime}
\end{array}\right.
$$

where $v=v(t, x)$ and $\sigma=\sigma(t, x)$ are time-dependent periodic functions on the line and the prime stands for the $x$-derivative. It can be viewed as a high-frequency limit of the 2-component Camassa-Holm equation, cf. [21].

It turns out that this system is closely related to the Kähler geometry of the Madelung transform and the Sasaki-Fisher-Rao metric (15). Consider the semi-direct product $\mathcal{G}=\operatorname{Diff}_{0}^{s+1}\left(S^{1}\right) \ltimes H^{s}\left(S^{1}, S^{1}\right)$, where $\operatorname{Diff}_{0}^{s+1}\left(S^{1}\right)$ is the group of circle diffeomorphisms that fix a prescribed point and $H^{s}\left(S^{1}, S^{1}\right)$ is the space of Sobolev $S^{1}$-valued maps of the circle. The group multiplication is given by

$$
(\varphi, \alpha) \cdot(\eta, \beta)=(\varphi \circ \eta, \beta+\alpha \circ \eta) .
$$

Define a right-invariant Riemannian metric on $\mathcal{G}$ at the identity element by

$$
\langle\langle(v, \sigma),(v, \sigma)\rangle\rangle_{\dot{H}^{1}}=\frac{1}{4} \int_{S^{1}}\left(\left(v^{\prime}\right)^{2}+\sigma^{2}\right) \mathrm{d} x .
$$


If $t \rightarrow(\varphi(t), \alpha(t))$ is a geodesic in $\mathcal{G}$ then $v=\dot{\varphi} \circ \varphi^{-1}$ and $\sigma=\dot{\alpha} \circ \varphi^{-1}$ satisfy equations (18). Lenells [13] showed that the map

$$
(\varphi, \alpha) \mapsto \sqrt{\varphi^{\prime} \mathrm{e}^{\mathrm{i} \alpha}}
$$

is an isometry from $\mathcal{G}$ to a subset of $\left\{\psi \in H^{s}\left(S^{1}, \mathbb{C}\right) \mid\|\psi\|_{L^{2}}=1\right\}$. Moreover, solutions to (18) satisfying $\int_{S^{1}} \sigma \mathrm{d} x=0$ correspond to geodesics in the complex projective space $\mathbb{P} H^{s}\left(S^{1}, \mathbb{C}\right)$ equipped with the Fubini-Study metric. Our results show that this isometry is a particular case of Theorem 3.3.

Proposition 3.7. The 2-component Hunter-Saxton equation (18) with initial data satisfying $\int_{S^{1}} \sigma \mathrm{d} x=0$ is equivalent to the geodesic equation of the Sasaki-Fisher-Rao metric (15) on $T^{*} \operatorname{Dens}^{s}\left(S^{1}\right)$.

Proof. First, observe that the mapping (20) can be rewritten as $(\varphi, \alpha) \mapsto$ $\boldsymbol{\Phi}(\pi(\varphi), \alpha)$, where $\boldsymbol{\Phi}$ is the Madelung transform and $\pi$ is the projection $\varphi \mapsto \varphi^{*} \mu$ specialized to the case $M=S^{1}$.

Next, observe that the metric (19) in the case $\int_{S^{1}} \sigma \mathrm{d} x=0$ is the pullback of the Sasaki metric (15) by the mapping

$$
\operatorname{Diff}_{0}^{s+1}\left(S^{1}\right) \ltimes H^{s}\left(S^{1}, S^{1}\right) \ni(\varphi, \alpha) \mapsto(\pi(\varphi),[\theta]) \in T^{*} \operatorname{Dens}^{s}\left(S^{1}\right),
$$

where $\theta(x)=\int_{0}^{x} \alpha^{\prime}(s) \mathrm{d} s$. Indeed, we have

$$
\begin{aligned}
\mathrm{G}_{(\pi(\varphi),[\theta])}^{*}\left(\frac{\mathrm{d}}{\mathrm{d} t} \pi(\varphi),[\dot{\alpha}]\right) & =\frac{1}{4} \int_{S^{1}}\left(\left(\frac{\dot{\varphi}^{\prime}}{\varphi^{\prime}}\right)^{2}+\dot{\alpha}^{2}\right) \varphi^{\prime} \mathrm{d} x \\
& =\frac{1}{4} \int_{S^{1}}\left(\left(\partial_{x}\left(\dot{\varphi} \circ \varphi^{-1}\right)\right)^{2}+\left(\dot{\alpha} \circ \varphi^{-1}\right)^{2}\right) \mathrm{d} x \\
& =\frac{1}{4} \int_{S^{1}}\left(\left(v^{\prime}\right)^{2}+\sigma^{2}\right) \mathrm{d} x .
\end{aligned}
$$

It follows from the change of variables formula by the diffeomorphism $\varphi$ that the condition $\int_{S^{1}} \sigma \mathrm{d} x=0$ corresponds to $\int_{S^{1}} \dot{\alpha} \varphi^{\prime} \mathrm{d} x=0$. Hence, the description of the 2-component Hunter-Saxton equation as a geodesic equation on the complex projective $L^{2}$ space is a special case of that on $T^{*} \operatorname{Dens}^{s}(M)$ with respect to the Sasaki-Fisher-Rao metric (15).

Remark 3.8. Observe that if $\sigma=0$ at $t=0$ then $\sigma(t)=0$ for all $t$ and the 2-component Hunter-Saxton equation (18) reduces to the standard HunterSaxton equation. This is a consequence of the fact that horizontal geodesics on $T^{*} \operatorname{Dens}^{s}(M)$ with respect to the Sasaki-Fisher-Rao metric descend to geodesics on Dens ${ }^{s}(M)$ with respect to the Fisher-Rao metric.

\section{Madelung transform as a momentum maP}

In $\S 2$ we described the Madelung transform as a symplectomorphism from $T^{*} \operatorname{Dens}(M)$ to $\mathbb{P} H^{s}(M, \mathbb{C} \backslash\{0\})$ which associates a wave function $\psi=$ $\sqrt{\rho} e^{\mathrm{i} \theta / 2}$ (modulo a phase factor $e^{\mathrm{i} \tau}$ ) to a pair $(\rho,[\theta])$ consisting of a density $\rho$ of unit mass and a function $\theta$ (modulo an additive constant). Here, we start by outlining (following [7]) another approach, which shows that it is 
natural to regard the inverse Madelung transform as a momentum map from the space $\mathbb{P} H^{s}(M, \mathbb{C})$ of wave functions $\psi$ to the set of pairs $(\rho \mathrm{d} \theta, \rho)$ regarded as elements of the dual space $\mathfrak{s}^{*}$ of a certain Lie algebra. The latter is a semidirect product $\mathfrak{s}=\mathfrak{X}(M) \ltimes H^{s}(M)$ corresponding to the Lie group $S=\operatorname{Diff}(M) \ltimes H^{s}(M)$. (In this section $\operatorname{Diff}(M)$ stands for Sobolev diffeomorphisms $\operatorname{Diff}^{s+1}(M)$ and $\mathfrak{X}(M)$ for vector fields $\mathfrak{X}^{s+1}(M)$.)

Furthermore, this construction generalizes to the vector-valued case $S_{(\ell)}=$ $\operatorname{Diff}(M) \ltimes H^{s}\left(M, \mathbb{C}^{\ell}\right)$. For $\ell=2$ this group appears naturally in the description of general compressible fluids including transport of both density and entropy. The case $\ell>1$ provides also a setting for quantum systems with spin degrees of freedom. For example, $\ell=2$ (a rank-1 spinor) describes fermions with spin $1 / 2$ (such as electrons, neutrons, and protons).

In $\S 4.7$ below we present a unifying point of view which explains the origin of the Madelung transform as the momentum map in a semidirect product reduction.

4.1. A group action on the space of wave functions. We start by defining a group action on the space of wave functions. First, observe that it is natural to think of $H^{s}(M, \mathbb{C})$ as a space of complex-valued half-densities on $M$. Indeed, $\psi \in H^{s}(M, \mathbb{C})$ is assumed to be square-integrable and $|\psi|^{2}$ is interpreted as a probability measure. Half-densities are characterized by how they are transformed under diffeomorphisms of the underlying space: the pushforward $\varphi_{*} \psi$ of a half-density $\psi$ on $M$ by a diffeomorphism $\varphi$ of $M$ is given by the formula

$$
\varphi_{*} \psi=\sqrt{\left|\operatorname{Det}\left(D \varphi^{-1}\right)\right|} \psi \circ \varphi^{-1} .
$$

This formula explains the following natural action of a semidirect product group on the vector space of half-densities.

Definition 4.1. [7] The semidirect product group $S=\operatorname{Diff}(M) \ltimes H^{s}(M)$ acts on the space $H^{s}(M, \mathbb{C})$ as follows: for a group element $(\varphi, a) \in S$ the action on wave functions $\psi$ is

$$
(\varphi, a) \circ \psi=\sqrt{\left|\operatorname{Det}\left(D \varphi^{-1}\right)\right|} e^{-\mathrm{i} a / 2}\left(\psi \circ \varphi^{-1}\right) .
$$

This action descends to the space of cosets $[\psi] \in \mathbb{P} H^{s}(M, \mathbb{C})$.

Thus, a wave function $\psi$ is pushed forward under the diffeomorphism $\varphi$ as a complex-valued half-density, followed by a pointwise phase adjustment by $e^{-\mathrm{i} a / 2}$. An easy computation gives the following Lie algebra action

Proposition 4.2. The infinitesimal action of $\xi=(v, \alpha) \in \mathfrak{s}$ on $H^{s}(M, \mathbb{C})$ corresponding to the action (21) is represented by the vector field $V_{\xi}$ on $H^{s}(M, \mathbb{C})$ defined at each point $\psi$ by

$$
V_{\xi}(\psi)=-\frac{1}{2} \psi \operatorname{div}(v)-\frac{\mathrm{i}}{2} \alpha \psi-\iota_{v} \mathrm{~d} \psi
$$


4.2. The inverse of the Madelung transform. Consider the following alternative definition of the inverse Madelung transform, which will be our primary object here. Let $\Omega^{1}(M)$ denote the space of 1 -forms on $M$ of Sobolev class $H^{s}$. Recall the definition (2) of the Madelung transform: $(\rho, \theta) \mapsto \psi=$ $\sqrt{\rho e^{i \theta}}$, where $\rho>0$.

Proposition 4.3. [7] The map

$$
\mathbf{M}: H^{s}(M, \mathbb{C}) \rightarrow \Omega^{1}(M) \times \operatorname{Dens}^{s}(M)
$$

given by

$$
\psi \mapsto(m, \rho)=(2 \operatorname{Im}(\bar{\psi} \mathrm{d} \psi), \bar{\psi} \psi)
$$

is the inverse of the Madelung transform (2) in the following sense: if $\psi=$ $\sqrt{\rho e^{\mathrm{i} \theta}}$ then $\mathbf{M}(\psi)=(\rho \mathrm{d} \theta, \rho)$.

Proof. For $\psi=\sqrt{\rho} e^{\mathrm{i} \theta / 2}$ one evidently has $\bar{\psi} \psi=\rho$. The expression for the other component follows from the observation

$$
\operatorname{Im} \bar{\psi} \mathrm{d} \psi=\bar{\psi} \psi \operatorname{Im} \mathrm{d}(\ln \psi)=\rho \operatorname{Im} \mathrm{d}((\ln \sqrt{\rho})+\mathrm{i} \theta / 2)=\rho \mathrm{d} \theta / 2 .
$$

These two components allow one to obtain $\rho$ and $\rho \mathrm{d} \theta$ and hence, by integration, to recover $\theta$ modulo an additive constant. (The ambiguity involving an additive constant in the definition of $\theta$ corresponds to recovering the wave function $\psi$ modulo a constant phase factor).

For a positive function $\rho$ satisfying $\int_{M} \rho \mu=1$ the pair $(\rho \mathrm{d} \theta, \rho)$ can be identified with $(\rho,[\theta])$ in $T^{*} \operatorname{Dens}(M)$, where the momentum variable $m=\rho \mathrm{d} \theta$ is naturally thought of as an element of $\mathfrak{X}(M)^{*}$. Note, however, that this definition of the inverse Madelung works in greater generality: the momentum variable $m$ is defined even when $\rho$ is allowed to be zero, although $\theta$ cannot be recovered there.

Remark 4.4. So far we viewed $\psi$ as a function on an $n$-manifold $M$. One can also consistently regard $\psi$ as a complex half-density $\varpi=\psi \mu^{1 / 2}$. The set of complex half-densities on $M$ is denoted $\sqrt{\Omega^{n}}(M) \otimes \mathbb{C}$ indicating that it is "the square root" of the space $\Omega^{n}(M)$ of $n$-forms. Then the map $\mathbf{M}$ in $(23)$ can be understood as follows. For a half-density $\varpi \in \sqrt{\Omega^{n}}(M) \otimes \mathbb{C}$ the second component $\bar{\varpi}$ of the map $\mathbf{M}$ is understood as a tensor product $(\bar{\psi} \psi) \mu=\rho \mu$ of two half-densities on $M$, thus yielding the density $\rho \in$ $\operatorname{Dens}^{s}(M)$. One can show that the first component $\operatorname{Im}(\varpi \mathrm{d} \varpi)$ of $\mathbf{M}$ can be regarded as an element $m \otimes \mu=\rho \mathrm{d} \theta \otimes \mu \in \Omega^{1}(M) \otimes_{H^{s}(M)} \Omega^{n}(M)$. Namely, given a reference density $\mu$, for any half-density $\varpi=f(x) \mu^{1 / 2}$ define its differential $\mathrm{d} \varpi:=\mathrm{d} f(x) \otimes \mu^{1 / 2}$. While the differential $\mathrm{d} \varpi$ depends on the choice of the reference density, the momentum map does not.

Proposition 4.5. For any half density $\varpi=f(x) \mu^{1 / 2} \in \sqrt{\Omega^{n}}(M) \otimes \mathbb{C}$ the momentum $2 \operatorname{Im}(\bar{\varpi} \mathrm{d} \varpi)=2 \operatorname{Im} \bar{f} \mathrm{~d} f \otimes \mu$ is a well defined element of $\Omega^{1}(M) \otimes_{H^{s}(M)} \Omega^{n}(M)$ and does not depend on the choice of the reference density $\mu$. 
Proof. Given a different reference volume form $\nu=h(x) \mu$ with a positive function $h>0$ one has $\varpi=f(x) \mu^{1 / 2}=g(x) \nu^{1 / 2}=g(x)(h(x) \mu)^{1 / 2}$, where $f(x)=g(x) \sqrt{h(x)}$ and

$$
\begin{aligned}
\operatorname{Im}(\bar{\varpi} \mathrm{d} \varpi) & =\operatorname{Im} \bar{f} \mathrm{~d} f \otimes \mu=\operatorname{Im} \bar{g} \sqrt{h} d(g \sqrt{h}) \otimes \mu \\
& =\operatorname{Im}(\bar{g} \sqrt{h} \sqrt{h} \mathrm{~d} g+\bar{g} \sqrt{h} g \mathrm{~d}(\sqrt{h})) \otimes \mu \\
& =\operatorname{Im} \bar{g} h \mathrm{~d} g \otimes \mu=\operatorname{Im} \bar{g} \mathrm{~d} g \otimes_{H^{s}(M)}(h \mu) \\
& =\operatorname{Im} \bar{g} \mathrm{~d} g \otimes \nu,
\end{aligned}
$$

where we dropped the term with $\bar{g} g \sqrt{h} \mathrm{~d}(\sqrt{h})$ since it is purely real.

Remark 4.6. The pair $(m, \rho) \otimes \mu=(\rho \mathrm{d} \theta \otimes \mu, \rho \mu)$ is understood as an element of the space $\mathfrak{s}^{*}=\Omega^{1}(M) \otimes_{H^{s}(M)} \Omega^{n}(M) \oplus \Omega^{n}(M)$ dual to the Lie algebra $\mathfrak{s}=\mathfrak{X}(M) \ltimes H^{s}(M)$, while the inverse Madelung transformation is a map $\mathbf{M}: H^{s}(M, \mathbb{C}) \rightarrow \mathfrak{s}^{*}$. Note that the dual space $\mathfrak{s}^{*}$ has a natural Lie-Poisson structure (as any dual Lie algebra).

4.3. A reminder on momentum maps. In the next section we show that the inverse Madelung transform (23) is a momentum map associated with the action (21) of the Lie group $S=\operatorname{Diff}(M) \ltimes H^{s}(M)$ on $H^{s}(M, \mathbb{C})$. We start by recalling the definition of a momentum map.

Suppose that a Lie algebra $\mathfrak{g}$ acts on a Poisson manifold $P$ and denote its action by $A: \mathfrak{g} \rightarrow \mathfrak{X}(P)$ where $A(\xi)=\xi_{P}$. Let $\langle$,$\rangle denote the pairing of \mathfrak{g}$ and $\mathfrak{g}^{*}$.

Definition 4.7. A momentum map associated with a Lie algebra action $A(\xi)=\xi_{P}$ is a map $\mathcal{M}: P \rightarrow \mathfrak{g}^{*}$ such that for every $\xi \in \mathfrak{g}$ the function $H_{\xi}: P \rightarrow \mathbb{R}$ defined by $H_{\xi}(p):=\langle\mathcal{M}(p), \xi\rangle$ for any $p \in P$ is a Hamiltonian of the vector field $\xi_{P}$ on the Poisson manifold $P$, i.e., $X_{H_{\xi}}(p)=\xi_{P}(p)$.

Thus, Lie algebra actions that admit momentum maps are Hamiltonian actions and the pairing of the momentum map at a point $p \in P$ with an element $\xi \in \mathfrak{g}$ defines a Hamiltonian function associated with the Hamiltonian vector field $\xi_{P}$ at that point $p$.

A momentum map $\mathcal{M}: P \rightarrow \mathfrak{g}^{*}$ of a Lie algebra $\mathfrak{g}$ is infinitesimally equivariant if for all $\xi, \eta \in \mathfrak{g}$ one has $H_{[\xi, \eta]}=\left\{H_{\xi}, H_{\eta}\right\}$, which means that not only for any Lie algebra vector defines a Hamiltonian vector field on the manifold, but also the Lie algebra bracket of two such fields corresponds to the Poisson bracket of their Hamiltonians.

4.4. Madelung transform is a momentum map. We now show (following Fusca [7]) that the transformation $\mathbf{M}$ is a momentum map associated with the action (22).

First note that the vector space $H^{s}(M, \mathbb{C}) \subset L^{2}(M, \mathbb{C})$ of Sobolev wave functions on $M$ is naturally equipped with the symplectic (and hence Poisson) structure $\{F, G\}(\psi)=\langle\langle\nabla F,-\mathrm{i} \nabla G\rangle\rangle_{L^{2}}=\langle\langle\mathrm{d} F, J \mathrm{~d} G\rangle\rangle_{L^{2}}$. This structure is related to the natural Hermitian inner product on $L^{2}(M, \mathbb{C}):\left\langle\langle f, g\rangle_{L^{2}}:=\right.$ 
$\int_{M} f \bar{g} \mu$ and the complex structure of multiplication by i. Now define the Hamiltonian function $H_{\xi}: H^{s}(M, \mathbb{C}) \rightarrow \mathbb{R}$ by $H_{\xi}(\psi):=\langle\mathbf{M}(\psi), \xi\rangle$.

Theorem 4.8. [7] For the Lie algebra $\mathfrak{s}=\mathfrak{X}(M) \ltimes H^{s}(M, \mathbb{R})$ its action (22) on the Poisson space $H^{s}(M, \mathbb{C}) \subset L^{2}(M, \mathbb{C})$ admits a momentum map. The inverse Madelung transformation $\mathbf{M}: H^{s}(M, \mathbb{C}) \rightarrow \mathfrak{s}^{*}$ defined by $(23)$ is, up to scaling by 4, a momentum map associated with this Lie algebra action.

Proof. The Hamiltonian vector field for the function $H_{\xi}$ is $X_{H_{\xi}}=-\mathrm{i} \mathrm{d} H_{\xi}$ where the differential is obtained from

$$
\left\langle\mathrm{d} H_{\xi}(\psi), \phi\right\rangle=\operatorname{Re}\left\langle\left\langle\mathrm{d} H_{\xi}(\psi), \phi\right\rangle\right\rangle_{L^{2}}=\left.\frac{\mathrm{d}}{\mathrm{d} t}\right|_{\epsilon=0} H_{\xi}(\psi+\epsilon \phi)
$$

for any $\phi$ in $H^{s}(M, \mathbb{C})$. Let $\xi=(v, \alpha)$ be an element of $\mathfrak{s}=\mathfrak{X}(M) \ltimes H^{s}(M)$ whose pairing with $(m, \rho) \in \mathfrak{s}^{*}$ is $\langle(v, \alpha),(m, \rho)\rangle:=\int_{M}(\rho \cdot \alpha+m \cdot v) \mu$. We have

$$
\begin{aligned}
H_{\xi}(\psi) & =\int_{M}\left(\mathbf{M}(\psi)^{\rho} \cdot \alpha+\mathbf{M}(\psi)^{m} \cdot v\right) \mu \\
& =\int_{M}\left(\bar{\psi} \psi \alpha+2 \operatorname{Im}\left(\iota_{v} \bar{\psi} \mathrm{d} \psi\right)\right) \mu \\
& =\operatorname{Re} \int_{M}\left(\bar{\psi} \psi \alpha-2 \mathrm{i} \iota_{v} \bar{\psi} \mathrm{d} \psi\right) \mu .
\end{aligned}
$$

To find the variational derivative let $\phi \in H^{s}(M, \mathbb{C})$ be a test function. Then

$$
\begin{aligned}
\left.\frac{d}{d \epsilon} H_{\xi}(\psi+\epsilon \phi)\right|_{\epsilon=0} & =\operatorname{Re} \int_{M}\left(\bar{\psi} \phi \alpha+\bar{\phi} \psi \alpha-2 \mathrm{i} \iota_{v} \bar{\phi} \mathrm{d} \psi-2 \mathrm{i} \iota_{v} \bar{\psi} \mathrm{d} \phi\right) \mu \\
& =\operatorname{Re} \int_{M}\left(2 \bar{\phi} \psi \alpha-2 \mathrm{i} \bar{\phi} \iota_{v} \mathrm{~d} \psi+2 \mathrm{i} \phi \operatorname{div}(\bar{\psi} v)\right) \mu \\
& =\operatorname{Re} \int_{M}\left(2 \bar{\phi} \psi \alpha+\overline{2 \mathrm{i} \phi \iota_{v} \mathrm{~d} \bar{\psi}}+2 \mathrm{i} \phi \bar{\psi} \operatorname{div}(v)+2 \mathrm{i} \phi \iota_{v} \mathrm{~d} \bar{\psi}\right) \mu \\
& =\operatorname{Re} \int_{M}\left(2 \psi \alpha-4 \mathrm{i} \iota_{v} \mathrm{~d} \psi-2 \mathrm{i} \psi \operatorname{div}(v)\right) \bar{\phi} \mu \\
& =\operatorname{Re}\left\langle\left\langle 2 \psi \alpha-4 \mathrm{i} \iota_{v} \mathrm{~d} \psi-2 \mathrm{i} \psi \operatorname{div}(v), \phi\right\rangle\right\rangle_{L^{2}},
\end{aligned}
$$

so that $\mathrm{d} H_{\xi}(\psi)=2 \psi \alpha-2 \mathrm{i} \psi \operatorname{div}(v)-4 \mathrm{i} \iota_{v} \mathrm{~d} \psi$. This implies that

$$
X_{H_{\xi}}(\psi)=-2 \mathrm{i} \alpha \psi-4 \iota_{v} \mathrm{~d} \psi-2 \psi \operatorname{div} v
$$

and comparing with (22) one obtains $X_{H_{\xi}}(\psi)=4 V_{\xi}(\psi)$.

Moreover, the Madelung transform turns out to be an infinitesimally equivariant momentum map, as was verified in [7]. (Recall that its equivariance means morphism of the Lie algebras: the Hamiltonian of the Lie bracket of two fields is the Poisson bracket of their Hamiltonians.) In particular, it follows that the Madelung transform is also a Poisson map taking the Poisson structure on $P$ (up to scaling by 4 ) to the Lie-Poisson structure on $\mathfrak{g}$, i.e., the map $\mathbf{M}: H^{s}(M, \mathbb{C}) \rightarrow \mathfrak{s}^{*}$ is infinitesimally equivariant for the action on $H^{s}(M, \mathbb{C})$ of the semidirect product Lie algebra $\mathfrak{s}$. This result is expected 
from the symplectomorphism result in Theorem 2.4 since $T^{*} \operatorname{Dens}^{s}(M)$ is a coadjoint orbit in $\mathfrak{s}^{*}$ via $(\rho,[\theta]) \mapsto(\rho \mathrm{d} \theta, \rho)$.

\subsection{Multi-component Madelung transform as a momentum map.}

There is a natural generalization of the above approach to the space of wave vector-functions $\psi \in H^{s}\left(M, \mathbb{C}^{\ell}\right)$, notably rank 1 spinors for which $\ell=2$. One needs to define an action of the group $S_{(\ell)}=\operatorname{Diff}(M) \ltimes H^{s}(M)^{\ell}$ on the subspace of smooth vector-functions.

Definition 4.9. The semidirect product group $S_{(\ell)}=\operatorname{Diff}(M) \ltimes H^{s}(M)^{\ell}$ acts on the space $\mathbb{P} H^{s}\left(M, \mathbb{C}^{\ell}\right)$ as follows: if $(\varphi, a) \in S_{(\ell)}$ is a group element, where $\varphi$ is a diffeomorphism, $\tilde{a}=\left(a_{1}, \ldots, a_{\ell}\right)$ is a vector, and $\tilde{\psi}=\left(\psi_{1}, \ldots, \psi_{\ell}\right)$ is a smooth wave vector-function, then

$$
\left(\varphi, a_{1}, \ldots, a_{\ell}\right): \psi_{k} \mapsto(\varphi, \tilde{a}) \circ \psi_{k}:=\sqrt{\left|\operatorname{Det}\left(D \varphi^{-1}\right)\right|} e^{-i a_{k} / 2}\left(\psi_{k} \circ \varphi^{-1}\right)
$$

for $k=1, \ldots, \ell$. The corresponding Lie algebra is denoted $\mathfrak{s}(\ell)$.

Definition 4.10. The (inverse) multicomponent Madelung transform is the $\operatorname{map} \mathbf{M}^{(\ell)}: H^{s}\left(M, \mathbb{C}^{\ell}\right) \rightarrow \mathfrak{s}_{(\ell)}^{*}$ defined by $\mathbf{M}^{(\ell)}(\tilde{\psi})=(m, \tilde{\rho})$, where $m=$ $2 \sum_{k=1}^{\ell} \operatorname{Im}\left(\bar{\psi}_{k} \mathrm{~d} \psi_{k}\right)$ and $\tilde{\rho}=\left(\rho_{1}, \ldots, \rho_{\ell}\right)$ with $\rho_{k}:=\bar{\psi}_{k} \psi_{k}$.

Here, as before, we have $m \otimes \mu \in \Omega^{1}(M) \otimes_{H^{s}(M)} \Omega^{n}(M)$ while for each $k=1, \ldots \ell$ we have $\rho_{k} \in H^{s}(M)$, so that $(m, \tilde{\rho}) \otimes \mu \in \mathfrak{s}_{(\ell)}^{*}$.

Similarly, the space $H^{s}\left(M, \mathbb{C}^{\ell}\right)$ has a natural symplectic (and hence Poisson) structure and one can prove a multicomponent version of Theorem 4.8.

Theorem 4.11. For the Lie algebra $\mathfrak{s}_{(\ell)}=\mathfrak{X}(M) \ltimes H^{s}(M)^{\ell}$, its action (24) on the Poisson space $H^{s}\left(M, \mathbb{C}^{\ell}\right)$ admits a momentum map. The map $\mathbf{M}^{(\ell)}: H^{s}\left(M, \mathbb{C}^{\ell}\right) \rightarrow \mathfrak{s}_{(\ell)}^{*}$ is a momentum map associated with this Lie algebra action. The Madelung transform is (up to scaling by 4) a Poisson map taking the bracket on $H^{s}\left(M, \mathbb{C}^{\ell}\right)$ to the Lie-Poisson bracket on the dual $\mathfrak{s}_{(\ell)}^{*}$ of the semidirect product Lie algebra $\mathfrak{s}_{(\ell)}$.

Remark 4.12. More generally, for any subgroup $G$ of $\mathrm{U}(\ell)$ one has an action on $H^{s}\left(M, \mathbb{C}^{\ell}\right)$ of the semidirect product $\widetilde{S}_{(\ell)}=\operatorname{Diff}(M) \ltimes H^{s}(M, G)$ of diffeomorphisms $\varphi$ with $G$-valued $H^{s}$-functions $A \in H^{s}(M, G)=H^{s}(M) \otimes G$ on $M$. It is given by

$$
(\varphi, A): \tilde{\psi} \mapsto \sqrt{\left|\operatorname{Det}\left(D \varphi^{-1}\right)\right|} A\left(\tilde{\psi} \circ \varphi^{-1}\right) .
$$

In particular, if $\ell=2$ (or $\ell=4$ ) the subgroup $G=\mathrm{SU}(\ell)$ acts by rotation of spinors (this may have some relevance for hydrodynamic formulations of the Pauli (or Dirac) equations). Note that the action of $\widetilde{S}_{(\ell)}$ preserves the Hermitian and symplectic structures on $H^{s}\left(M, \mathbb{C}^{\ell}\right)$ and admits a momentum map.

Remark 4.13. From the viewpoint of Hamiltonian dynamics specifying a larger $\ell$ (and considering the corresponding semi-direct product groups $S_{(\ell)}$ ) 
corresponds to "exploring a larger chunk" of the phase space $T^{*} \operatorname{Diff}(M) \simeq$ $\operatorname{Diff}(M) \times \mathfrak{X}(M)^{*}$ (cf. next section). Indeed, for $\ell=1$ the corresponding equations on $T^{*} \operatorname{Dens}^{s}(M)$ only allows for momenta of the form $m=\rho \mathrm{d} \theta$ (corresponding to potential-type solutions of the barotropic Euler equations). By choosing $\ell>0$ we allow for momenta of the form $m=\sum_{k=1}^{\ell} \rho_{k} \mathrm{~d} \theta_{k}$ thus filling out a larger portion of $\mathfrak{X}(M)^{*}$.

4.6. Example: general compressible fluids. For general compressible (nonbarotropic) inviscid fluids the equation of state describes the pressure as a function $P(\rho, \sigma)$ of both density $\rho$ and entropy $\sigma$. Thus, the corresponding equations of motion include the evolution of all three quantities: the velocity $v$ of the fluid, its density $\rho$ and the entropy $\sigma$.

$$
\left\{\begin{array}{l}
\dot{v}+\nabla_{v} v+\frac{1}{\rho} \nabla P(\rho, \sigma)=0, \\
\dot{\rho}+\operatorname{div}(\rho v)=0 \\
\dot{\sigma}+\mathcal{L}_{v} \sigma=0
\end{array}\right.
$$

In the case the entropy is constant or the pressure is independent of $\sigma$ this system describes a barotropic flow, see equations (9). Note that, while the density evolves as an $n$-form, the entropy evolves as a function. However, according to the continuity equation, passing to the entropy density $\varsigma=\sigma \rho$ one can regard the corresponding group as the semidirect product $S_{(2)}=$ $\operatorname{Diff}(M) \ltimes\left(H^{s}(M) \oplus H^{s}(M)\right)$, which leads to a Hamiltonian picture on the dual $\mathfrak{s}_{(2)}^{*}$. By applying the multicomponent Madelung transform $\mathbf{M}^{(2)}$ one can rewrite and interpret this system on the space of rank- 1 spinors $H^{s}\left(M, \mathbb{C}^{2}\right)$. Indeed, the evolution of the momentum $m \otimes \mu=v^{b} \otimes \mu$ is

$$
\dot{m} \otimes \mu+\mathcal{L}_{v}(m \otimes \mu)+\mathrm{d} \frac{\delta U}{\delta \rho} \otimes \rho \mu+\mathrm{d} \frac{\delta U}{\delta \varsigma} \otimes \varsigma \mu=0 .
$$

Observe that an invariant subset of solutions is given by those with momenta $m=\rho \mathrm{d} \theta+\varsigma \mathrm{d} \tau$, where $\theta, \tau \in H^{s}(M) / \mathbb{R}$. They can be regarded as analogs of potential solutions of the barotropic fluid equations. We thereby obtain a canonical set of equations on $T^{*} H^{s}(M)^{2}$ given by

$$
\begin{cases}\dot{\rho}=\frac{\delta H}{\delta \theta} & \dot{\varsigma}=\frac{\delta H}{\delta \tau} \\ \dot{\theta}=-\frac{\delta H}{\delta \rho} & \dot{\tau}=-\frac{\delta H}{\delta \varsigma}\end{cases}
$$

for a Hamiltonian of the form

$$
H(\rho, \varsigma, \theta, \tau)=\frac{1}{2} \int_{M}\left(|\nabla \theta|^{2} \rho+|\nabla \tau|^{2} \varsigma\right) \mu+U(\rho, \varsigma) .
$$

Using the multicomponent Madelung transform

$$
(\rho, \varsigma, \theta, \tau) \mapsto\left(\sqrt{\rho} \mathrm{e}^{\mathrm{i} \theta / 2}, \sqrt{\varsigma} \mathrm{e}^{\mathrm{i} \tau / 2}\right),
$$

and Theorem 4.11 this gives (up to scaling by 4) a Hamiltonian system for the spinor $\tilde{\psi}=\left(\psi_{1}, \psi_{2}\right) \in H^{s}\left(M, \mathbb{C}^{2}\right)$ with the Hamiltonian given by

$$
H(\tilde{\psi})=\frac{1}{2}\|\nabla \tilde{\psi}\|_{L^{2}}^{2}+W\left(\left|\psi_{1}\right|^{2},\left|\psi_{2}\right|^{2}\right) .
$$


for the potential

$$
W(\rho, \varsigma)=-\frac{1}{2}\|\nabla \sqrt{\rho}\|_{L^{2}}^{2}-\frac{1}{2}\|\nabla \sqrt{\varsigma}\|_{L^{2}}^{2}+\frac{1}{4} U(\rho, \varsigma),
$$

where the functional $U$ is related to the pressure function $P(\rho, \sigma)$ of the compressible Euler equation. The corresponding Schrödinger equation reads

$$
\mathrm{i} \dot{\tilde{\psi}}=-\Delta \tilde{\psi}+\left(\begin{array}{cc}
\frac{\delta W}{\delta \rho} & 0 \\
0 & \frac{\delta W}{\delta \varsigma}
\end{array}\right) \tilde{\psi} .
$$

Conversely, one can work backwards to obtain a fluid formulation of various quantum-mechanical spin Hamiltonians, such as the Pauli equations for spin $1 / 2$ particles of a given charge.

4.7. Geometry of semi-direct product reduction. In this section we present the geometric structure behind the semi-direct product reduction which reveals the origin of the Madelung transform as the moment map above.

Let $N$ be a Lie subgroup of a Lie group $G$. Assume that $G$ acts from the left on a linear space $V$ (a left representation of $G$ ). The quotient space of left cosets $G / N$ is acted upon from the left by $G$. Assume now that $G / N$ can be embedded as an orbit in $V$ and let $\gamma: G / N \rightarrow V$ denote the embedding. Since the action of $G$ on $V$ induces a linear left dual action on $V^{*}$ we can construct the semi-direct product $S=G \ltimes V^{*}$.

Proposition 4.14. The quotient $T^{*} G / N$ is naturally embedded via a Poisson map in the Lie-Poisson space $\mathfrak{s}^{*}$ (the dual of the corresponding semidirect product algebra).

Proof. The Poisson embedding is given by

$$
([g], m) \mapsto(m, \gamma([g]))
$$

where we use that $T^{*} G / N \simeq G / N \times \mathfrak{g}^{*}$ and $\mathfrak{s}^{*} \simeq \mathfrak{g}^{*} \times V$. Now, the action of $S$ on $\mathfrak{s}^{*}$ is

$$
(g, a) \cdot(m, b)=\operatorname{Ad}_{(g, a)}^{*}(m, b)=\left(\operatorname{Ad}_{g}^{*}(m)-\mathcal{M}(a, b), g \cdot b\right),
$$

where $\mathcal{M}: V^{*} \times V \rightarrow \mathfrak{g}^{*}$ is the momentum map associated with the cotangent lifted action of $G$ on $V^{*}$. The corresponding infinitesimal action of $\mathfrak{s}$ is

$$
(\xi, \dot{a}) \cdot(m, b)=\operatorname{ad}_{(\xi, \dot{a})}^{*}(m, b)=\left(\operatorname{ad}_{\xi}^{*}(m)-\mathcal{M}(\dot{a}, b), \xi \cdot b\right) .
$$

Since the second component is only acted upon by $g$ (or $\xi$ ), but not $a$ (or $\dot{a})$, it follows from the embedding of $G / N$ as an orbit in $V$ that we have a natural Poisson action of $S$ (or $\mathfrak{s}$ ) on $T^{*} G / N$ via the Poisson embedding (26). Notice that the momentum map of $S$ (or $\mathfrak{s}$ ) acting on $\mathfrak{s}^{*}$ is the identity: this follows since the Hamiltonian vector field on $\mathfrak{s}^{*}$ for $H(m, b)=\langle m, \xi\rangle+\langle b, \dot{a}\rangle$ is given by (28). 
We now return to the standard symplectic reduction (without semi-direct products). The dual $\mathfrak{n}^{*}$ of the subalgebra $\mathfrak{n} \subset \mathfrak{g}$ is naturally identified with affine cosets of $\mathfrak{g}^{*}$ such that

$$
m \in\left[m_{0}\right] \Longleftrightarrow\left\langle m-m_{0}, \xi\right\rangle=0 \quad \forall \xi \in \mathfrak{n} .
$$

The momentum map of the subgroup $N$ acting on $\mathfrak{g}^{*}$ by $\mathrm{Ad}^{*}$ is then given by $m \mapsto[m]$, since the momentum map of $G$ acting on $\mathfrak{g}^{*}$ is the identity. If $\langle m, \mathfrak{n}\rangle=0$, i.e., $m \in(\mathfrak{g} / \mathfrak{n})^{*}$, then $m \in[0]$ is in the zero momentum coset. Since we also have $T^{*}(G / N) \simeq G / N \times(\mathfrak{g} / \mathfrak{n})^{*}$ this gives us an embedding as a symplectic leaf in $T^{*} G / N \simeq G / N \times \mathfrak{g}^{*}$. The restriction to this leaf is called zero-momentum symplectic reduction.

Turning to the semi-direct product reduction, we now have Poisson embeddings of $T^{*}(G / N)$ in $T^{*} G / N$ and of $T^{*} G / N$ in $\mathfrak{s}^{*}$. The combined embedding of $T^{*}(G / N)$ as a symplectic leaf in $\mathfrak{s}^{*}$ is given by the map

$$
([g], a) \mapsto(\mathcal{M}(a, \gamma([g])), \gamma([g]))
$$

This implies that we have a Hamiltonian action of $S$ (or $\mathfrak{s})$ on the zeromomentum symplectic leaf $T^{*}(G / N)$ sitting inside $T^{*} G / N$, which in turn sits inside $\mathfrak{s}^{*}$.

Since $S$ has a natural symplectic action on $\mathfrak{s}^{*}$ and since $G / N$ is an orbit in $V \simeq V^{* *}$, we have, by restriction, a natural action of $S$ on $T^{*} G / N$. Furthermore, since the momentum map associated with $S$ acting on $\mathfrak{s}^{*}$ is the identity, the Poisson embedding map (26) is the momentum map for $S$ acting on $T^{*} G / N$. Thus, the momentum map of $S$ acting on $T^{*}(G / N)$ is given by $(30)$.

The above considerations are summarized in the following theorem.

Theorem 4.15. The inverse of the Madelung transform viewed as a momentum map (Section 4.4) can be regarded as the semi-direct product reduction and a Poisson embedding $T^{*}(G / N) \rightarrow \mathfrak{s}^{*}$ as described above for the groups $G=\operatorname{Diff}(M)$ and $N=\operatorname{Diff}_{\mu}(M)$.

\section{Appendix A. The FUnCtional-AnALYTiC SETting}

The infinite-dimensional geometric constructions in this paper can be rigorously carried out in any reasonable function space setting in which the topology is at least as strong as $C^{1}$, satisfies the functorial axioms of Palais [18] and admits a Hodge decomposition. The choice of the Sobolev spaces is very convenient for the purposes of this paper because many of the technical details which were used (explicitly or implicitly) in the proofs can be readily traced in the literature. We briefly review the main points below.

As introduced in the main body of the paper the notation $\operatorname{Diff}^{s}(M)$ stands for the completion of the group of smooth $C^{\infty}$ diffeomorphisms of an $n$ dimensional compact Riemannian manifold $M$ with respect to the $H^{s}$ topology where $s>n / 2+1$. This puts the Sobolev lemma at our disposal and thus equipped $\operatorname{Diff}^{s}(M)$ becomes a smooth Hilbert manifold whose tangent 
space at the identity $T_{e} \operatorname{Diff}^{s}(M)$ consists of all $H^{s}$ vector fields on $M$, see e.g., [6], Section 2.

Using the implicit function theorem the subgroup $\operatorname{Diff}_{\mu}^{s}(M)=\{\eta \in$ $\left.\operatorname{Diff}^{s}(M): \eta^{*} \mu=\mu\right\}$ consisting of those diffeomorphisms that preserve the Riemannian volume form $\mu$ can then be shown to inherit the structure of a smooth Hilbert submanifold with $T_{e} \operatorname{Diff}_{\mu}^{s}(M)=\left\{v \in T_{e} \operatorname{Diff}^{s}: \operatorname{div} v=0\right\}$, cf. e.g., [6], Sections 4 and 8.

Standard results on compositions and products of Sobolev functions ensure that both Diff ${ }^{s}$ and $\operatorname{Diff}_{\mu}^{s}$ are topological groups with right translations $\xi \rightarrow \xi \circ \eta$ (resp., left translations $\xi \rightarrow \eta \circ \xi$ and inversions $\xi \rightarrow \xi^{-1}$ ) being smooth (resp., continuous) as maps in the $H^{s}$ topology, cf. [18]; Chapters 4 and 9. Furthermore, the natural projection

$$
\pi: \operatorname{Diff}^{s+1}(M) \rightarrow \operatorname{Diff}^{s+1}(M) / \operatorname{Diff}_{\mu}^{s+1}(M) \simeq \operatorname{Dens}^{s}(M)
$$

given by $\eta \rightarrow \pi(\eta)=\eta^{*} \mu$ extends to a smooth submersion between $\operatorname{Diff}^{s+1}(M)$ and the space of right cosets which can be identified with the space of probability densities on $M$ of Sobolev class $H^{s}$ (cf. Section 2 above). More technical details, as well as proofs of all these facts, can be found in $[6,18]$ and their bibliographies.

\section{Appendix B. A comment on Rescaling Constants}

First, recall that for any $(\dot{\rho},[\dot{\theta}]) \in T_{(\rho,[\theta])} T^{*} \operatorname{Dens}^{s}(M)$ we can pick a representative $\dot{\theta} \in[\dot{\theta}]$ such that $\int_{M} \dot{\theta} \rho \mu=0$. The canonical symplectic structure on $T^{*}$ Dens $^{s}$ is then given by

$$
\Omega_{(\rho,[\theta])}\left(\left(\dot{\rho}_{1}, \dot{\theta}_{1}\right),\left(\dot{\rho}_{2}, \dot{\theta}_{2}\right)\right)=\int_{M}\left(\dot{\theta}_{2} \dot{\rho}_{1}-\dot{\theta}_{1} \dot{\rho}_{2}\right) \mu .
$$

Furthermore, for any $\alpha>0$ we have a complex structure

$$
J_{(\rho,[\theta])}(\dot{\rho}, \dot{\theta})=\left(-\alpha \dot{\theta} \rho, \frac{1}{\alpha} \frac{\dot{\rho}}{\rho}\right) .
$$

Combining the two structures in a standard manner yields a Kähler metric on $T^{*} \operatorname{Dens}^{s}(M)$

$$
\begin{aligned}
\mathrm{G}_{(\rho,[\theta])}^{*}\left(\left(\dot{\rho}_{1}, \dot{\theta}_{1}\right),\left(\dot{\rho}_{2}, \dot{\theta}_{2}\right)\right) & =\Omega_{(\rho,[\theta])}\left(\left(\dot{\rho}_{1}, \dot{\theta}_{1}\right), J_{(\rho,[\theta])}\left(\dot{\rho}_{2}, \dot{\theta}_{2}\right)\right) \\
& =\int_{M}\left(\frac{1}{\alpha} \frac{\dot{\rho}_{1} \dot{\rho}_{2}}{\rho}+\alpha \dot{\theta}_{1} \dot{\theta}_{2} \rho\right) \mu .
\end{aligned}
$$

Next, we turn to the Madelung transform which, for a fixed constant $\gamma \neq 0$, is

$$
\psi=\sqrt{\rho \mathrm{e}^{\mathrm{i} \theta / \gamma}}
$$

and whose derivative is

$$
\dot{\psi}=\frac{\psi}{2}\left(\frac{\dot{\rho}}{\rho}+\frac{\mathrm{i}}{\gamma} \dot{\theta}\right) .
$$


Given $\beta>0$ the (scaled) Fubini-Study Hermitian structure on $\mathbb{P} C^{\infty}(M, \mathbb{C})$ is given by

$$
\mathrm{FS}_{\psi}\left(\dot{\psi}_{1}, \dot{\psi}_{2}\right)=\beta \frac{\left\langle\left\langle\dot{\psi}_{1}, \dot{\psi}_{2}\right\rangle\right\rangle_{L^{2}}}{\langle\langle\psi, \psi\rangle\rangle_{L^{2}}}-\beta \frac{\left\langle\left\langle\dot{\psi}_{1}, \psi\right\rangle\right\rangle_{L^{2}}\left\langle\left\langle\psi, \dot{\psi}_{2}\right\rangle\right\rangle_{L^{2}}}{\langle\langle\psi, \psi\rangle\rangle_{L^{2}}^{2}}
$$

where $\langle\langle\phi, \psi\rangle\rangle_{L^{2}}=\int_{M} \phi \bar{\psi} \mu$. If $\dot{\psi}_{1}, \dot{\psi}_{2}$ are of the form (32), then it follows from $\int_{M} \rho \mu=1$ and $\int_{M} \dot{\rho}_{i} \mu=\int_{M} \dot{\theta}_{i} \rho \mu=0$ that $\|\psi\|_{L^{2}}=1$ and $\left\langle\left\langle\dot{\psi}_{i}, \psi\right\rangle\right\rangle_{L^{2}}=0$. In this case

$$
\begin{aligned}
\mathrm{FS}_{\psi}\left(\dot{\psi}_{1}, \dot{\psi}_{2}\right) & =\beta\left\langle\left\langle\dot{\psi}_{1}, \dot{\psi}_{2}\right\rangle\right\rangle_{L^{2}}=\frac{\beta}{4} \int_{M}|\psi|^{2}\left(\frac{\dot{\rho}_{1}}{\rho}+\frac{\mathrm{i}}{\gamma} \dot{\theta}_{1}\right)\left(\frac{\dot{\rho}_{1}}{\rho}-\frac{\mathrm{i}}{\gamma} \dot{\theta}_{1}\right) \mu \\
& =\frac{\beta}{4 \gamma} \int_{M}\left\{\left(\gamma \frac{\dot{\rho}_{1}}{\rho} \dot{\rho}_{2}+\frac{\dot{\theta}_{1} \dot{\theta}_{2}}{\gamma} \rho\right)-\mathrm{i}\left(\dot{\theta}_{2} \dot{\rho}_{1}-\dot{\theta}_{1} \dot{\rho}_{2}\right)\right\} \mu
\end{aligned}
$$

and the associated symplectic structure is

$$
\begin{aligned}
\hat{\Omega}_{\psi}\left(\dot{\psi}_{1}, \dot{\psi}_{2}\right) & =\operatorname{ReFS}_{\psi}\left(\mathrm{i} \dot{\psi}_{1}, \dot{\psi}_{2}\right)=-\operatorname{ImFS} \mathrm{FS}_{\psi}\left(\dot{\psi}_{1}, \dot{\psi}_{2}\right) \\
& =\frac{\beta}{4 \gamma} \int_{M}\left(\dot{\theta}_{2} \dot{\rho}_{1}-\dot{\theta}_{1} \dot{\rho}_{2}\right) \mu=\frac{\beta}{4 \gamma} \Omega_{(\rho,[\theta])}\left(\dot{\rho}_{1}, \dot{\theta}_{1}, \dot{\rho}_{2}, \dot{\theta}_{2}\right) .
\end{aligned}
$$

Thus, the Madelung transform as defined by (31) is a symplectomorphism up to a rescaling by the constant $\beta / 4 \gamma$.

Similarly, the Riemannian metric associated with (33) is

$$
\begin{aligned}
\hat{\mathrm{G}}_{\psi}^{*}\left(\dot{\psi}_{1}, \dot{\psi}_{2}\right) & =\operatorname{ReFS}_{\psi}\left(\dot{\psi}_{1}, \dot{\psi}_{2}\right) \\
& =\frac{\beta}{4 \gamma} \int_{M}\left(\gamma \frac{\dot{\rho}_{1} \dot{\rho}_{2}}{\rho}+\frac{\dot{\theta}_{1} \dot{\theta}_{2}}{\gamma} \rho\right) \mu .
\end{aligned}
$$

Thus, to make the Madelung transform defined by (31) an isometry (up to rescaling by $\beta / 4 \gamma$ ) we require that $\alpha=\gamma$. If, in addition, it is to be a Kähler morphism, then we also require $\beta=4 \gamma$. Note that in this paper we set $\gamma=1$ while in his original work Madelung used $\gamma=\hbar / 2$ (as did von Renesse [20]).

\section{REFERENCES}

[1] V. I. Arnold, Sur la géométrie différentielle des groupes de Lie de dimension infinie et ses applications à l'hydrodynamique des fluides parfaits, Ann. Inst. Fourier (Grenoble) 16 (1966), 319-361.

[2] V. I. Arnold and B. A. Khesin, Topological Methods in Hydrodynamics, vol. 125 of Applied Mathematical Sciences, Springer-Verlag, New York, 1998.

[3] M. Bauer, M. Bruveris, and P. W. Michor, Uniqueness of the FisherRao metric on the space of smooth densities, Bulletin of the London Mathematical Society 48 (2016), 499-506.

[4] J. W. Bush, Quantum mechanics writ large, Proc. Natl. Acad. Sci. USA 107 (2010), 17455-17456. 
[5] Y. Couder, S. Protiere, E. Fort, and A. Boudaoud, Dynamical phenomena: Walking and orbiting droplets, Nature 437 (2005), 208.

[6] D. G. Ebin and J. E. Marsden, Groups of diffeomorphisms and the notion of an incompressible fluid., Ann. of Math. 92 (1970), 102-163.

[7] D. Fusca, The Madelung transform as a momentum map, J. Geom. Mech. 9 (2017), 157-165.

[8] H. Hasimoto, A soliton on a vortex filament, Journal of Fluid Mechanics 51 (1972), 477-485.

[9] B. Khesin, J. Lenells, G. Misiołek, and S. C. Preston, Geometry of diffeomorphism groups, complete integrability and geometric statistics, Geom. Funct. Anal. 23 (2013), 334-366.

[10] B. Khesin, G. Misiolek, and K. Modin, Geometric hydrodynamics via Madelung transform, Proc. Natl. Acad. Sci. USA 115 (2018), 61656170 .

[11] T. W. B. Kibble, Geometrization of quantum mechanics, Comm. Math. Phys. 65 (1979), 189-201.

[12] J. Langer and R. Perline, Poisson geometry of the filament equation, $J$. Nonlinear Sci. 1 (1991), 71-93.

[13] J. Lenells, Spheres, Kähler geometry and the Hunter-Saxton system, Proceedings of the Royal Society A: Mathematical, Physical and Engineering Sciences 469 (2013), 20120726-20120726.

[14] E. Madelung, Quantentheorie in hydrodynamischer form, Zeitschrift für Physik 40 (1927), 322-326.

[15] M. Molitor, On the relation between geometrical quantum mechanics and information geometry, J. Geom. Mech. 7 (2015), 169-202.

[16] E. Nelson, Review of stochastic mechanics, Journal of Physics: Conference Series 361 (2012), 012011.

[17] F. Otto, The geometry of dissipative evolution equations: the porous medium equation, Comm. Partial Differential Equations 26 (2001), 101-174.

[18] R. S. Palais, Foundations of global non-linear analysis, Benjamin, New York, 1968.

[19] B. N. Shashikanth, Vortex dynamics in $\mathbb{R}^{4}$, Journal of Mathematical Physics 53 (2012), 013103.

[20] M.-K. von Renesse, An optimal transport view of Schrödinger's equation, Canad. Math. Bull 55 (2012), 858-869.

[21] H. Wu and M. Wunsch, Global existence for the generalized twocomponent Hunter-Saxton system, Journal of Mathematical Fluid Mechanics 14 (2011), 455-469. 
Department of Mathematics, University of Toronto, Toronto, ON M5S 2E4, CANADA

E-mail address: khesin@math.toronto.edu

Department of Mathematics, University of Notre Dame, Notre Dame, iN 46556, USA

E-mail address: gmisiole@nd.edu

Department of Mathematical Sciences, Chalmers University of Technology And University of Gothenburg, SE-412 96 Gothenburg, Sweden

E-mail address: klas.modin@chalmers.se 